\title{
THE DISTORTION THEOREMS OF LINEAR INVARIANT FAMILY ON THE UNIT BALL*
}

\section{SHENG GONG ${ }^{\dagger}$ AND QIHUANG YU}

1. Introduction. It is well know that the distortion theorem of biholomorphic mapping in any domain in $\mathbb{C}^{n}$ does not exist. It exists only when the mapping class has more restrictions.

The first affirmative result was established by Barnard, FitzGerald and Gong[1] in 1988(The paper was published at 1994). They proved the following result.

A linear invarient family, hereafter denoted LIF, on the unit ball $\boldsymbol{B}^{n}$ in $\mathbb{C}^{n}$ is a family $\mathcal{M}$ of localy biholomorphic mappings $f: \boldsymbol{B}^{n} \rightarrow \mathbb{C}^{n}$ such that if $f \in \mathcal{M}$ then (i) $f(0)=0, J_{f}(0)=I$, where $J_{f}$ is the Jacobi matrix of $f$ and (ii) $\Lambda_{\varphi}$ of $f \in \mathcal{M}$ for $\varphi \in \operatorname{Aut}\left(\boldsymbol{B}^{n}\right)$, the holomorphic automorphisms of $\boldsymbol{B}^{n}$. Here $\Lambda_{\varphi}(f)$ is the Koebe transform of $f, \Lambda_{\varphi}(f)=\left(J_{\varphi}(0)\right)^{-1}\left(J_{f}(\varphi(0))^{-1}[f(\varphi(z))-f(\varphi(0))]\right.$.

Let $\mathcal{S}$ be a linear invariant family, on the unit ball $\boldsymbol{B}^{2}$ in $\mathbb{C}^{2}$. If $f(z)=\left(f_{1}(z), f_{2}(z)\right)$ $\in \mathcal{S}, z=\left(z_{1}, z_{2}\right) \in \boldsymbol{B}^{2}$, and

$$
\left\{\begin{array}{l}
f_{1}(z)=z_{1}+d_{2,0}^{(1)} z_{1}^{2}+d_{1,1}^{(1)} z_{1} z_{2}+d_{0,2}^{(1)} z_{2}^{2}+\cdots \cdots \\
f_{2}(z)=z_{2}+d_{2,0}^{(2)} z_{1}^{2}+d_{1,1}^{(2)} z_{1} z_{2}+d_{0,2}^{(2)} z_{2}^{2}+\cdots \cdots
\end{array}\right.
$$

then

$$
\left|\log \left[\left(1-z \bar{z}^{\prime}\right)^{\frac{3}{2}} \operatorname{det} J_{f}(z)\right]\right| \leq C(\mathcal{S}) \log \frac{1+|z|}{1-|z|}
$$

holds, where $C(\mathcal{S})=\operatorname{Sup}\left\{\left|d_{2,0}^{(1)}+\frac{1}{2} d_{1,1}^{(2)}\right| \mid f \in \mathcal{S}\right\}, J_{f}(z)$ is the Jacobi matrix of $f$ at $z$.

From (1.1), it follows the following distortion theorem

$$
\frac{(1-|z|)^{C(\mathcal{S})-\frac{3}{2}}}{(1+|z|)^{C(\mathcal{S})+\frac{3}{2}}} \leq\left|\operatorname{det} J_{f}(z)\right| \leq \frac{(1+|z|)^{C(\mathcal{S})-\frac{3}{2}}}{(1-|z|)^{C(\mathcal{S})+\frac{3}{2}}} .
$$

In particular, if $\mathcal{S}$ is the family of normalized biholomorphic convex mapping, then one can prove $1.5 \leq C(\mathcal{S}) \leq 1.761$.

In 1989 , at his doctor thesis, Liu[11] extended this result to $\mathbb{C}^{n}$. He proved that: Let $\mathcal{S}$ be a LIF on the unit ball $\boldsymbol{B}^{n}$ in $\mathbb{C}^{n}$. If

$$
f(z)=\left(f_{1}(z), f_{2}(z), \cdots \cdots, f_{n}(z)\right)=z+\left(z A^{(1)} z^{\prime}, \cdots \cdots, z A^{(n)} z^{\prime}\right)+\cdots \cdots \in \mathcal{S}
$$

where $A^{(i)}=\left(a_{j k}^{(i)}\right)_{1 \leq j, k \leq n}, z \in \boldsymbol{B}^{n}$, then

$$
\left|\log \left[\left(1-z \bar{z}^{\prime}\right)^{\frac{n+1}{2}} \operatorname{det} J_{f}(z)\right]\right| \leq \log \frac{1+|z|}{1-|z|},
$$

\footnotetext{
${ }^{*}$ Received June 20, 2000; accepted for publication October 23, 2000. Suppoted by National Science Foundation of China.

${ }^{\dagger}$ Department of Mathematics, University of Science and Technology of China, Hefei, Anhui, 230026, China (gongs@sina.com).

$\ddagger$ Institute of Applied Mathematics, Academia Sinica, Beijing, 100080, China.
} 
where

$$
C(\mathcal{S})=\operatorname{Sup}\left\{\mid \sum_{i=1}^{n} a_{i 1}^{(i)} \| f \in \mathcal{S}\right\} .
$$

As a consequence, we have

$$
\frac{(1-|z|)^{C(\mathcal{S})-\frac{n+1}{2}}}{(1+|z|)^{C(\mathcal{S})+\frac{n+1}{2}}} \leq\left|\operatorname{det} J_{f}(z)\right| \leq \frac{(1+|z|)^{C(\mathcal{S})-\frac{n+1}{2}}}{(1-|z|)^{C(\mathcal{S})+\frac{n+1}{2}}}
$$

He also proved that if $\mathcal{S}$ is the family of normalized biholomorphic convex mappings, then

$$
\frac{n+1}{2} \leq C(\mathcal{S}) \leq 1+\frac{\sqrt{2}(n-1)}{2} .
$$

It was conjectured by Barnard, FitzGerald and Gong[1] that $C(\mathcal{S})=\frac{n+1}{2}$, where $\mathcal{S}$ is the family of biholomorphic convex mappings.

In 1997, Pfaltzgraff [12] proved (1.6) again. The proof of Pfaltzgraff is same as Liu's proof in his doctor thesis.

In 1999, Pfaltzgraff and Suffridge [13] gave a counterexample to disprove the conjecture : $C(\mathcal{S})=\frac{n+1}{2}$ when $\mathcal{S}$ is the family of normalized convex mappings. Until now, nobody know the exact value of $C(\mathcal{S})$ when $\mathcal{S}$ is the family of normalized convex mappings, even nobody conjecture it.

In 1990, Gong and Zheng [8] extended (1.4) and (1.6) to the bounded symmetric domains.

Let $M \subset \mathbb{C}^{n}$ be a bounded symmetric domain containing the origin. Let $G$ be the group of holomorphic automorphism of $M$, and let $K$ be isotropy subgroup in $G$ which fixes the origin. Let $\mathcal{G}$ be Lie algebla of $G, \mathcal{K}$ be the maximal compact subalgebla of $\mathcal{G}$ corresponding to $K$. Then $\mathcal{G}$ has the Cartan decomposition.

$$
\mathcal{G}=\mathcal{K}+\mathcal{P}
$$

Suppose $\mathcal{A}$ is a maximal Abelian subalgebla in $\mathcal{P}$ and $\mathrm{A}$ is the corresponding analytic subgroup in $G$. Then we have the Iwasawa decomposition $G=K A N$. For every $z \in M$, there exist $X \in \mathcal{A}$ and $k \in K$ such that

$$
z=\xi(k a \cdot O)=\xi(\exp A d(k) X \cdot O)
$$

where $O=e K$ is the identity coset in $G / K$ and $\xi$ is the holomorphic diffeomorphism from $G / K$ onto $M$. If $X_{1}, \cdots \cdots, X_{q}$ form a basis of $\mathcal{A}$, then $X=\sum_{j=1}^{q} x_{j} X_{j}$, where $q=\operatorname{dim} \mathcal{A}=\operatorname{rank} M$. If $\mathcal{S}$ is a LIF on $M$, and $f(z) \in \mathcal{S}$, then

$$
\left|\log \frac{\operatorname{det} J_{f}(z)}{\sqrt{K(z, \bar{z}) / K(0,0)}}\right| \leq C(\mathcal{S}) \sum_{p=1}^{q} \log \frac{1+\left|\tanh x_{p}\right|}{1-\left|\tanh x_{p}\right|}
$$

holds, where $K(z, \bar{z})$ is the Bergman kernel function on $M, C(\mathcal{S})$ is defined by (1.5) when $f(z)$ has expression (1.3).

In 1993, Gong Wang and Yu [6] established the following result. If $f(z)$ is a normalized convex mapping on the unit ball $\boldsymbol{B}^{n}$ in $\mathbb{C}^{n}$, then

$$
\left(\frac{1-|z|}{1+|z|}\right)^{2} G \leq J_{f}(z) \overline{J(z)}^{\prime} \leq\left(\frac{1+|z|}{1-|z|}\right)^{2} G
$$


holds, where

$$
G=\left(g_{i j}\right)_{1 \leq i, j \leq n}=\left(\frac{\left(1-|z|^{2}\right) \delta_{i j}+\overline{z_{i}} z_{j}}{\left(1-|z|^{2}\right)^{2}}\right)_{1 \leq i, j \leq n}
$$

is the matrix of the Bergman metic of $\boldsymbol{B}^{n}$.

From (1.9), we immediately have the following consequences:

$$
\frac{(1-|z|)^{n-1}}{(1+|z|)^{3 n+1}} \leq \operatorname{det}\left(J_{f}{\overline{J_{f}}}^{\prime}\right) \leq \frac{(1+|z|)^{n-1}}{(1-|z|)^{3 n+1}} .
$$

Comparing (1.11) with (1.6) and (1.7), we can see that (1.6) and (1.7) is better then (1.11).

$$
\frac{1}{(1+|z|)^{2}} \leq\left\|J_{f}(z)\right\| \leq \frac{1}{(1-|z|)^{2}}
$$

where $\left\|J_{f}(z)\right\|$ is the norm of the matrix $J_{f}(z)$.

$$
\frac{1-|z|}{(1+|z|)^{3}} \leq \Lambda_{n}^{2} \leq \frac{1+|z|}{(1-|z|)^{3}}
$$

where $\Lambda_{n}^{2}$ is the smallest eigenvalue of $J_{f}(z){\overline{J_{f}(z)}}^{\prime}$.

$$
\frac{1}{(1+|z|)^{4}}+\frac{(n-1)(1-|z|)}{(1+|z|)^{3}} \leq \operatorname{Tr}\left(J_{f}(z){\overline{J_{f}(z)}}^{\prime}\right) \leq \frac{1}{(1-|z|)^{4}}+\frac{(n-1)(1+|z|)}{(1-|z|)^{3}} \text {. }
$$

In 1999, Gong and Liu [5] extended (1.9) to bounded convex circular domains.

Let $\Omega$ be a bounded convex circular domain in $\mathbb{C}^{n}, 0 \in \Omega$, its Minkowski functional $p(z) \in \mathcal{C}^{1}$ on $\bar{\Omega}$ except for a lower dimensional manifold. Let $f(z)$ be a normalized biholomorphic convex mapping on $\Omega$, then

$$
\frac{1-p(z)}{1+p(z)} F(z, \xi) \leq p\left(J_{f}(z) \xi\right) \leq \frac{1+p(z)}{1-p(z)} F(z, \xi)
$$

holds, where $\xi \in \mathbb{C}^{n}$ is a column vector, $F(z, \xi)=F_{c}(z, \xi)=F_{k}(z, \xi), F_{c}(z, \xi)$ is the Carathéodory metric in infinitesimal form and $F_{k}(z, \xi)$ is the Kobayashi-Royden metric in infinitesimal form.(By Lempert Theorem [10], $F_{c}$ and $F_{k}$ are same when the domain is convex.) When $\Omega$ is unit ball $\boldsymbol{B}^{n}$ in $\mathbb{C}^{n}$, then [10]

$$
F(z, \xi)=F_{c}(z, \xi)=F_{k}(z, \xi)=\frac{|\xi|^{2}}{1-|z|^{2}}+\frac{\xi \bar{z}^{\prime} z \bar{\xi}^{\prime}}{\left(1-|z|^{2}\right)}
$$

In this case, (1.15) becomes (1.9).

When $\xi=z$ in $(1.15)$, we have

$$
\frac{p(z)}{(1+p(z))^{2}} \leq p\left(J_{f}(z) z\right) \leq \frac{p(z)}{(1-p(z))^{2}}
$$

where $z \in \mathbb{C}^{n}$ is a column vector. It implies

$$
\frac{1-p(z)}{(1+p(z))^{2}} \leq\left|\lambda_{n}(z)\right| \leq \cdots \cdots \leq\left|\lambda_{1}(z)\right| \leq \frac{1+p(z)}{(1-p(z))^{2}}
$$


where $\left|\lambda_{1}(z)\right|^{2} \geq \cdots \cdots \geq\left|\lambda_{n}(z)\right|^{2}$ are eigenvalues of $J_{f}(z) \overline{J_{f}(z)}$.

In the case that $\Omega$ is the unit ball $\boldsymbol{B}^{n}$ in $\mathbb{C}^{n}$, we compare (1.17) with (1.12) and (1.13), we may find that (1.12) and (1.13) are better than (1.17).

Let $f(z)$ be a normalized holomorphic mapping on a domain $\Omega$ in $\mathbb{C}^{n}$, and $0 \in \Omega$. We may expand $f(z)$ at $z=0$ as

$$
f(z)=z+\sum_{j=2}^{\infty} \frac{1}{j !} D^{j} f(0)(z, \cdots \cdots, z),
$$

where $D^{j}$ is the Frechet derivative of order $j, j=1,2, \cdots \ldots$. If $\Omega$ is a bounded symmetric domain in $\mathbb{C}^{n}$, and $\mathcal{S}$ is a LIF, then we may find that $C(\mathcal{S})$ defined by (1.5) can express as

$$
C(\mathcal{S})=\operatorname{Sup}\left\{\frac{1}{2}\left|\operatorname{Tr}\left(D^{2} f(0) u\right)\right| \mid u \bar{u}^{\prime}=1, f \in \mathcal{S}\right\} .
$$

Recently, Pfaltzgraff and Suffridge [14] define another order. If $\mathcal{S}$ is a LIF on a domain $\Omega$ which containing origin, they defined

$$
\alpha=\|\operatorname{ord}\| \mathcal{S}=\operatorname{Sup}\left\{\frac{1}{2}\left\|D^{2} f(0)\right\| \mid f \in \mathcal{S}\right\} .
$$

They proved that if $\Omega$ is the unit ball in $\mathbb{C}^{n}, \mathcal{S}$ is a LIF, $f \in \mathcal{S}$, then

$$
\frac{(1-|z|)^{\alpha-1}}{(1+|z|)^{\alpha+1}} \leq\|D f(z)\| \leq \frac{(1+|z|)^{\alpha-1}}{(1-|z|)^{\alpha+1}},
$$

where $\alpha$ is the order of $\mathcal{S}$ which is defined by (1.20).

Hamada and Kohr [9] used same method as Pfaltzgraff and Suffridge used in [14] to prove the similar result when $\Omega$ is the unit polydisk in $\mathbb{C}^{n}$.

There are many examples of LIF at [14]. When LIF is the family of normalized convex mapping on $\boldsymbol{B}^{n}$, then $\alpha=1,(1.21)$ becomes (1.12). All normalized localy biholomorphic mappings on $\mathbb{C}^{n}$ form a LIF, then $\alpha=\infty$ for this family.

As we mentioned above, we gave the estimations of $\left|\operatorname{det} J_{f}(z)\right|$ by (1.6) and (1.7), and the estimations of $\left\|J_{f}(z)\right\|$ by (1.21) already, it is natural to ask the estimations of $\operatorname{Tr}\left(J_{f}(z){\overline{J_{f}(z)}}^{\prime}\right)$. In the Section 2, we will give the estimations of $\operatorname{Tr}\left(J_{f}(z){\overline{J_{f}(z)}}^{\prime}\right)$ for LIF on the unit ball $\boldsymbol{B}^{n}$ in $\mathbb{C}^{n}$ by (1.20). When LIF is the family of normalized convex mappings, it is coincided with (1.14). In Section 3, by using the method as we used in Section 2, we may prove (1.6) by a different approach. As another application of (1.9), we will give the estimations of the covariant derivative of biholomorphic convex mappings on the unit ball in Section 4.

2. The estimations of the trace of Jacobi matrix of mappings in a $B^{n}$. In this section, all vectors are column vectors. We consider the LIF on the unit ball $\boldsymbol{B}^{n}$ in $\mathbb{C}^{n}$. We already know that [14]:

(1) $\|$ ord $\| \mathcal{S} \geq 1$ for every LIF $\mathcal{S}$. $\|\operatorname{ord}\| \mathcal{S}=1$ if $\mathcal{S}$ is the family of normalized biholomorphic convex mappings on $\boldsymbol{B}^{n}$.

(2) If $\mathcal{S}$ is a LIF and $\|$ ord $\| \mathcal{S}=\alpha<\infty$, then $\mathcal{S}$ is a normal family.

(3) If $\varphi_{a}(z) \in \operatorname{Aut}\left(\boldsymbol{B}^{n}\right)$, which maps 0 to $a \in \boldsymbol{B}^{n}$, and

$$
f(z ; a)=\left(D \varphi_{a}(0)\right)^{-1}\left(D f\left(\varphi_{a}(0)\right)^{-1}\left[f\left(\varphi_{a}(z)\right)-f\left(\varphi_{a}(0)\right)\right]\right.
$$


where $f \in \mathcal{S}$, a LIF. Then the definition of $\|$ ord $\| \mathcal{S}$ can be expressed as

$$
\|\operatorname{ord}\| \mathcal{S}=\operatorname{Sup}\left\{\frac{1}{2}\left|D^{2} f(0 ; a)(w, w)\right||f \in \mathcal{S},| w|=1,| a \mid<1\right\} .
$$

(4) $C(\mathcal{S}) \leq n \|$ ord $\| \mathcal{S}$, when $\mathcal{S}$ is a LIF on $\boldsymbol{B}^{n}$.

We have the estimations of $\left|\operatorname{det} J_{f}(z)\right|$ by (1.6) and the estimations of $\left\|J_{f}(z)\right\|$ by (1.21) already. In this section, we will give the estimations of $\left.\operatorname{Tr}\left(J_{f}(z) \overline{J_{f}(z)}\right)^{\prime}\right)$ when $f$ belongs to a LIF $\mathcal{S}$ with $\|$ ord $\| \mathcal{S}=\alpha$ on the unit ball $\boldsymbol{B}^{n}$ in $\mathbb{C}^{n}$.

THEOREM 2.1 If $\mathcal{S}$ is a LIF on the unit ball $\boldsymbol{B}^{n}$ in $\mathbb{C}^{n}$ with $\|$ ord $\| \mathcal{S}=\alpha$, then

$$
\begin{aligned}
& \frac{(1-|z|)^{2 \alpha-2}}{(1+|z|)^{2 \alpha+2}}+(n-1) \frac{(1-|z|)^{2 \alpha-1}}{(1+|z|)^{2 \alpha+1}} \\
\leq & \operatorname{Tr}\left(J_{f}(z){\overline{J_{f}(z)}}^{\prime}\right) \leq \frac{(1+|z|)^{2 \alpha-2}}{(1-|z|)^{2 \alpha+2}}+(n-1) \frac{(1+|z|)^{2 \alpha-1}}{(1-|z|)^{2 \alpha+1}}
\end{aligned}
$$

for all $f \in \mathcal{S}$ and $z \in \boldsymbol{B}^{n}$.

The inequalities (1.21) are the estimations of the largest eigenvalue of $J_{f}(z){\overline{J_{f}(z)}}^{\prime}$. Theorem 2.1 tells us that there exists one largest eigenvalue only in $J_{f}(z){\overline{J_{f}}(z)}^{\prime}$, all other eigenvalues have lower order comparing with this largest eigenvalue if the largest eigenvalue of $J_{f}(z){\overline{J_{f}(z)}}^{\prime}$ attend the estimating order.

When $\mathcal{S}$ is the family of normalized biholomorphic convex mappings, then $\|$ ord $d \| \mathcal{S}$ $=1,(2.3)$ is coincide with (1.14).

If $S$ is a LIF on the unit polydisc on $\mathbb{C}^{n}$, then we can get the similar conclusion, but we omit to state the results and its proof. The proof is similar with the proof of Theorem 2.1 in below.

The method of the proof of Theorem 2.1 is different but relatyed with the proof of (1.21) by Pfaltzgraff and Suffridge [14]. In purpose to prove Theorm 2.1, we need the following lemmas.

LEMMA 2.2 Fix $z_{0}=r_{0} u_{0} \in \boldsymbol{B}^{n}, u_{0} \in \partial \boldsymbol{B}^{n}$. If $F$ is the extremal mapping in $\mathcal{S}$ for the following maximum problem:

$$
\operatorname{Tr}\left({\overline{J_{F}\left(z_{0}\right)}}^{\prime} J_{F}\left(z_{0}\right)\right) \geq \operatorname{Tr}\left({\overline{J_{f}\left(z_{0}\right)}}^{\prime} J_{f}\left(z_{0}\right)\right)
$$

holds for any $f \in \mathcal{S}$, then

$$
\operatorname{Tr}\left({\overline{J_{F}\left(z_{0}\right)}}^{\prime} J_{F}\left(z_{0}\right)\right)=\max \left\{\operatorname{Tr}\left(\overline{J_{F}(z)} J_{F}(z)\right)|| z \mid=r_{0}\right\} .
$$

Proof. If (2.4) were false, then there would be a point $z_{1} \neq z_{0},\left|z_{1}\right|=r_{0}$ such that

$$
\operatorname{Tr}\left({\overline{J_{F}\left(z_{1}\right)}}^{\prime} J_{F}\left(z_{1}\right)\right)>\operatorname{Tr}\left(\overline{J_{F}\left(z_{0}\right)} J_{F}\left(z_{0}\right)\right) .
$$

There exists an unitary matrix $U$ such that $U z_{0}=z_{1}$ where $z_{0}, z_{1}$ are column vectors. Consider $F_{U}(z)=U^{-1} F(U z) \in \mathcal{S}$, then $J_{F_{U}}(z)=U^{-1} J_{F}(U z) U$, and consequently

$$
\operatorname{Tr}\left({\overline{J_{F_{U}}(z)}}^{\prime} J_{F_{U}}(z)\right)=\operatorname{Tr}\left({\overline{J_{F}(U z)}}^{\prime} J_{F}(U z)\right)
$$


which yieds the contradiction

$$
\operatorname{Tr}\left(\overline{J_{F_{U}}\left(z_{0}\right)^{\prime}} J_{F_{U}}\left(z_{0}\right)\right)=\operatorname{Tr}\left({\overline{J_{F}\left(z_{1}\right)}}^{\prime} J_{F}\left(z_{1}\right)\right)>\operatorname{Tr}\left(\overline{J_{F}\left(z_{0}\right)} J_{F}\left(z_{0}\right)\right)
$$

LeMma 2.3 Fix $z_{0}=r_{0} u_{0} \in \boldsymbol{B}^{n}, u_{0} \in \partial \boldsymbol{B}^{n}$. If $F$ is the extremal mapping in $\mathcal{S}$ for the maximum problem in Lemma 2.2, then $\operatorname{Tr}\left[D^{2} F\left(z_{0}\right) z_{0} \overline{J_{F}\left(z_{0}\right)}{ }^{\prime}\right]$ is real, equivalently, $\operatorname{Tr}\left[D^{2} F\left(z_{0}\right) u_{0}{\overline{J_{F}\left(z_{0}\right)}}^{\prime}\right]$ is real.

Proof. Let $t$ be a small real number, then

$$
J_{F}\left(z_{0} e^{i t}\right)=J_{F}\left(z_{0}+i t z_{0}+O\left(t^{2}\right)\right)=J_{F}\left(z_{0}\right)+i t D^{2} F\left(z_{0}\right) z_{0}+O\left(t^{2}\right) .
$$

We have

(2.5)

${\overline{J_{F}\left(z_{0} e^{i t}\right)}}^{\prime} J_{F}\left(z_{0} e^{i t}\right)={\overline{J_{F}\left(z_{0}\right)}}^{\prime} J_{F}\left(z_{0}\right)-i t{\overline{D^{2} F\left(z_{0}\right) z_{0}}}^{\prime} J_{F}\left(z_{0}\right)+i t D^{2} F\left(z_{0}\right) z_{0} \overline{J_{F}\left(z_{0}\right)}+O\left(t^{2}\right)$.

By Lemma 2.2,

$$
\begin{aligned}
0 & \geq \operatorname{Tr}\left(\overline{J_{F}\left(z_{0} e^{i t}\right)} J_{F}\left(z_{0} e^{i t}\right)\right)-\operatorname{Tr}\left(\overline{J_{F}\left(z_{0}\right)} J_{F}\left(z_{0}\right)\right) \\
& =i t \operatorname{Tr}\left(D^{2} F\left(z_{0}\right) z_{0}{\overline{J_{F}\left(z_{0}\right)}}^{\prime}-J_{F}\left(z_{0}\right){\overline{D^{2} F\left(z_{0}\right) z_{0}}}^{\prime}\right)+O\left(t^{2}\right) \\
& =2 \Re e \operatorname{Tr}\left(i t D^{2} F\left(z_{0}\right) z_{0}{\overline{J_{F}\left(z_{0}\right)}}^{\prime}\right)+O\left(t^{2}\right)
\end{aligned}
$$

Dividing by $|t|$ and considering both $t \rightarrow 0^{+}$and $t \rightarrow 0^{-}$, we have

$$
\operatorname{Im}\left\{\operatorname{Tr}\left(D^{2} F\left(z_{0}\right) z_{0}{\overline{J_{F}\left(z_{0}\right)}}^{\prime}\right\}=0\right. \text {. }
$$

LemMA 2.4. Fix $z_{0}=r_{0} u_{0} \in \boldsymbol{B}^{n}, u_{0} \in \partial \boldsymbol{B}^{n}$. If $F$ is the extremal mapping in $\mathcal{S}$ for the maximum problem in Lemma 2.2, then

$$
\begin{aligned}
& \operatorname{Tr}\left\{{\overline{J_{F}\left(z_{0}\right)}}^{\prime} D^{2} F\left(z_{0}\right) \nu\right\}-\operatorname{Tr}\left\{{\overline{J_{F}\left(z_{0}\right)}}^{\prime} D^{2} F\left(z_{0}\right) z_{0}{\overline{z_{0}}}^{\prime} \nu\right\} \\
- & \operatorname{Tr}\left\{{\overline{z_{0}}}^{\prime}{\overline{J_{F}\left(z_{0}\right)}}^{\prime} J_{F}\left(z_{0}\right) \nu\right\}-\operatorname{Tr}\left\{J_{F}\left(z_{0}\right){\overline{J_{F}\left(z_{0}\right)}}^{\prime}{\overline{z_{0}}}^{\prime} \nu\right\}-\operatorname{Tr}\left\{J_{F}\left(z_{0}\right){\overline{J_{F}\left(z_{0}\right)}}^{\prime} D^{2} F(0) \nu\right\} \\
= & 0
\end{aligned}
$$

holds for any $\nu \in \partial \boldsymbol{B}^{n}$, where $z_{0}, \nu$ are column vectors.

Proof. Any $f \in \mathcal{S}$ can expand as (1.18), it can express as

$$
f(z)=z+A_{2}(z, z)+A_{3}(z, z, z)+\cdots \cdots
$$

also. The mapping defined by (2.1) belongs to $\mathcal{S}$ and can be expanded as [14]

$$
f(z ; a)=f(z)+D f(z)\left(a-\bar{a}^{\prime} z z\right)-a-2 A_{2}(a, f(z))+O\left(|a|^{2}\right),
$$

where $|a|$ is sufficiently small. Let $D F(z ; a)$ be the Fréchet derivative of $F(z ; a)$ with respect to $z$, then [14]

$D F(z ; a)=J_{F}(z)+D^{2} F(z)\left(a-\bar{a}^{\prime} z z\right)-J_{F}(z) z \bar{a}^{\prime}-\bar{a}^{\prime} z J_{F}(z)-D^{2} F(0) a J_{F}(z)+O\left(|a|^{2}\right)$, 
when $|a|$ is sufficiently small, and

$$
\begin{aligned}
& \overline{D F(Z ; a)}^{\prime} D F(z ; a) \\
& ={\overline{J_{F}(z)}}^{\prime} J_{F}(z)+\left[D^{2} F(z)\left(a-\bar{a}^{\prime} z z\right)-J_{F}(z) z \bar{a}^{\prime}-\bar{a}^{\prime} z J_{F}(z)-D^{2} F(0) a J_{F}(z)\right]{\overline{J_{F}(z)}}^{\prime} \\
& +J_{F}(z)\left[{\overline{D^{2} F(z) a}}^{\prime}-z \bar{a}^{\prime}{\overline{D^{2} F(z) z}}^{\prime}-a \bar{z}^{\prime}{\overline{J_{F}(z)}}^{\prime}-{\overline{J_{F}(Z)}}^{\prime} \bar{z}^{\prime} a-{\overline{J_{F}(z)}}^{\prime}{\overline{D^{2} F(0) a}}^{\prime}\right] \\
& +O\left(|a|^{2}\right) \text {. }
\end{aligned}
$$

By the extremal property of $F$ at point $z_{0}$, we have

$$
\begin{aligned}
r l 0 & \geq \operatorname{Tr}\left\{{\overline{D F\left(z_{0} ; a\right)}}^{\prime} D F\left(z_{0} ; a\right)\right\}-\operatorname{Tr}\left({\overline{J_{F}\left(z_{0}\right)}}^{\prime} J_{F}\left(z_{0}\right)\right) \\
& =2 \Re e\left[\operatorname{Tr}\left[D^{2} F(z) a{\overline{J_{F}(z)}}^{\prime}\right]-\operatorname{Tr}\left[\bar{a}^{\prime} z D^{2} F(z) z{\overline{J_{F}(z)}}^{\prime}\right]\right. \\
& \left.-\operatorname{Tr}\left[J_{F}(z) a \bar{z}^{\prime}{\overline{J_{F}(z)}}^{\prime}\right]-\operatorname{Tr}\left[\bar{z}^{\prime} a J_{F}(z){\overline{J_{F}(z)}}^{\prime}\right]-\operatorname{Tr}\left[D^{2} F(0) a J_{F}(z){\overline{J_{F}(z)}}^{\prime}\right]\right]+O\left(|a|^{2}\right) .
\end{aligned}
$$

By Lemma 2.3, we know that $\operatorname{Tr}\left[D^{2} F\left(z_{0}\right) z_{0} \overline{J_{F}\left(z_{0}\right)}\right]$ is real, thus

$$
\Re e\left[\operatorname{Tr}\left\{\bar{a}^{\prime} z_{0} D^{2} F\left(z_{0}\right) z_{0}{\overline{J_{F}\left(z_{0}\right)}}^{\prime}\right\}\right]=\Re e\left[\operatorname{Tr}\left\{{\overline{z_{0}}}^{\prime} a D^{2} F\left(z_{0}\right) z_{0}{\overline{J_{F}\left(z_{0}\right)}}^{\prime}\right\}\right] .
$$

Substituting it into the previous inequality, we have

$$
\begin{aligned}
0 \geq & \Re e\left[\operatorname{Tr}\left[D^{2} F\left(z_{0}\right) a{\overline{J_{F}\left(z_{0}\right)}}^{\prime}\right]-\operatorname{Tr}\left[{\overline{z_{0}}}^{\prime} a D^{2} F\left(z_{0}\right) z_{0}{\overline{J_{F}\left(z_{0}\right)^{\prime}}}^{\prime}\right]\right. \\
& -\operatorname{Tr}\left[J_{F}\left(z_{0}\right) a{\overline{z_{0}}}^{\prime}{\overline{J_{F}\left(z_{0}\right.}}^{\prime}\right]-\operatorname{Tr}\left[{\overline{z_{0}}}^{\prime} a J_{F}\left(z_{0}\right){\overline{J_{F}\left(z_{0}\right)}}^{\prime}\right] \\
& \left.-\operatorname{Tr}\left[D^{2} F(0) a J_{F}\left(z_{0}\right){\overline{J_{F}\left(z_{0}\right)}}^{\prime}\right]\right]+O\left(|a|^{2}\right) .
\end{aligned}
$$

Dividing $|a|$, and denoting $\nu=\frac{a}{|a|}$, then let $|a| \rightarrow 0$, note that $a$ can be replaced by $e^{i t} a$ with arbitrary real $t$. We have proved (2.6).

LEMMA 2.5. Fix $z_{0}=r_{0} u_{0} \in \boldsymbol{B}^{n}, u_{0} \in \partial \boldsymbol{B}^{n}$. If $F$ is the extremal mapping in $\mathcal{S}$ for the maximum problem in Lemma 2.2, then

$$
\begin{aligned}
& \frac{\operatorname{Tr}\left({\overline{J_{F}(z)}}^{\prime} J_{F}(z)\right)-\operatorname{Tr}\left(\overline{J_{F}\left(z_{0}\right)} J_{F}\left(z_{0}\right)\right)}{2\left(r-r_{0}\right)}{ }^{\prime} \\
= & \frac{r_{0}}{1-r_{0}^{2}}\left[\operatorname{Tr}\left(J_{F}\left(z_{0}\right)^{\prime J_{F}\left(z_{0}\right)}\right)+\operatorname{Tr}\left({\overline{u_{0}}}^{\prime}{\overline{J_{F}\left(z_{0}\right)}}^{\prime} J_{F}\left(z_{0}\right) u_{0}\right)\right] \\
+ & \frac{1}{1-r_{0}^{2}} \operatorname{Tr}\left(J_{F}\left(z_{0}\right){\overline{J_{F}\left(z_{0}\right)}}^{\prime} D^{2} F(0) u_{0}\right)+O\left(\left|r-r_{0}\right|\right),
\end{aligned}
$$

where $z=r u_{0}$, and $r$ is a real number near $r_{0}$.

Proof. We expand $J_{F}(z)$ at point $z_{0}$,

$$
J_{F}(z)=J_{F}\left(z_{0}+z-z_{0}\right)=J_{F}\left(z_{0}\right)+D^{2} F\left(z_{0}\right)\left(z-z_{0}\right)+O\left(\left|z-z_{0}\right|^{2}\right) .
$$

Thus

$$
\begin{aligned}
{\overline{J_{F}(z)}}^{\prime} J_{F}(z)= & {\overline{J_{F}\left(z_{0}\right)}}^{\prime} J_{F}\left(z_{0}\right)+J_{F}\left(z_{0}\right){\overline{D^{2} F\left(z_{0}\right)\left(z-z_{0}\right)}}^{\prime} \\
& +D^{2} F\left(z_{0}\right)\left(z-z_{0}\right){\overline{J_{F}\left(z_{0}\right)}}^{\prime}+O\left(\left|z-z_{0}\right|^{2}\right) .
\end{aligned}
$$


We have

$$
\begin{aligned}
& \operatorname{Tr}\left(\overline{J_{F}(z)}{ }^{\prime} J_{F}(z)\right)-\operatorname{Tr}\left({\overline{J_{F}\left(z_{0}\right)}}^{\prime} J_{F}\left(z_{0}\right)\right) \\
= & 2 \Re e\left[\operatorname{Tr}\left(D^{2} F\left(z_{0}\right)\left(z-z_{0}\right){\overline{J_{F}\left(z_{0}\right)}}^{\prime}\right)\right]+O\left(\left|z-z_{0}\right|^{2}\right) .
\end{aligned}
$$

Dividing $2\left(r-r_{0}\right)$ on both sides, it is

$$
\begin{aligned}
& \frac{\operatorname{Tr}\left(\overline{J_{F}(z)}{ }^{\prime} J_{F}(z)\right)-\operatorname{Tr}\left(\overline{J_{F}\left(z_{0}\right)^{\prime}} J_{F}\left(z_{0}\right)\right)}{2\left(r-r_{0}\right)} \\
= & \Re e\left[\operatorname{Tr}\left(D^{2} F\left(z_{0}\right) u_{0} \overline{J_{F}\left(z_{0}\right)}\right)\right]+O\left(\left|r-r_{0}\right|\right) .
\end{aligned}
$$

By Lemma 2.3, we know that

$$
\Re e\left[\operatorname{Tr}\left(D^{2} F\left(z_{0}\right) u_{0}{\overline{J_{F}\left(z_{0}\right)}}^{\prime}\right)\right]=\operatorname{Tr}\left(D^{2} F\left(z_{0}\right) u_{0}{\overline{J_{F}\left(z_{0}\right)}}^{\prime}\right) .
$$

Thus

$$
\frac{\operatorname{Tr}\left({\overline{J_{F}(z)}}^{\prime} J_{F}(z)\right)-\operatorname{Tr}\left({\overline{J_{F}\left(z_{0}\right)}}^{\prime} J_{F}\left(z_{0}\right)\right)}{2\left(r-r_{0}\right)}=\operatorname{Tr}\left(D^{2} F\left(z_{0}\right) u_{0}{\overline{J_{F}\left(z_{0}\right)}}^{\prime}\right)+O\left(\left|r-r_{0}\right|\right) .
$$

Let $\nu=u_{0}$ in Lemma 2.4, (2.6) become

$$
\begin{aligned}
& \operatorname{Tr}\left\{{\overline{J_{F}\left(z_{0}\right)}}^{\prime} D^{2} F\left(z_{0}\right) u_{0}\right\}-r_{0}^{2} \operatorname{Tr}\left\{{\overline{J_{F}\left(z_{0}\right)}}^{\prime} D^{2} F\left(z_{0}\right) u_{0}\right\} \\
- & \operatorname{Tr}\left\{{\overline{z_{0}}}^{\prime}{\overline{J_{F}\left(z_{0}\right)}}^{\prime} J_{F}\left(z_{0}\right) u_{0}\right\}-\operatorname{Tr}\left\{J_{F}\left(z_{0}\right){\overline{J_{F}\left(z_{0}\right)}}^{\prime} u_{0}\right\}-\operatorname{Tr}\left\{J_{F}\left(z_{0}\right){\overline{J_{F}\left(z_{0}\right)}}^{\prime} D^{2} F(0) u_{0}\right\} \\
= & 0 .
\end{aligned}
$$

Hence

$$
\begin{aligned}
& \operatorname{Tr}\left(D^{2} F\left(z_{0}\right) u_{0}{\overline{J_{F}\left(z_{0}\right)}}^{\prime}\right) \\
= & \frac{r_{0}}{1-r_{0}^{2}}\left[\operatorname{Tr}\left[J_{F}\left(z_{0}\right){\overline{J_{F}\left(z_{0}\right.}}^{\prime}\right]+\operatorname{Tr}\left[{\overline{u^{\prime}}}^{\prime} \overline{J_{F}\left(z_{0}\right)} J_{F}\left(z_{0}\right) u_{0}\right]\right] \\
+ & \frac{1}{1-r_{0}^{2}} \operatorname{Tr}\left[J_{F}\left(z_{0}\right){\overline{J_{F}\left(z_{0}\right)^{\prime}}}^{\prime} D^{2} F(0) u_{0}\right] .
\end{aligned}
$$

Substituting (2.11) into (2.10), we get (2.8).

We now proceed with the proof of Theorem 2.1, beginning with the upper bound.

Proof of Theorem 2.1. The upper bound:Throughout the proof of the upper bound, $F$ will be the extremal mapping for the maximum problem associated with a fixed point $z_{0} \in \boldsymbol{B}^{n}$, i.e.,

$$
\operatorname{Tr}\left(\overline{J_{F}\left(z_{0}\right)} J_{F}\left(z_{0}\right)\right) \geq \operatorname{Tr}\left(\overline{J_{f}\left(z_{0}\right)} J_{f}\left(z_{0}\right)\right)
$$

holds for all $f \in \mathcal{S}$. We now assume that the upper bound in (2.3) is false. Hence there exists $r_{0} \in(0,1), z_{0} \in \boldsymbol{B}^{n},\left|z_{0}\right|=r_{0}$ such that

$$
\operatorname{Tr}\left(\overline{J_{F}\left(z_{0}\right)} J_{F}^{\prime}\left(z_{0}\right)\right)>\frac{\left(1+r_{0}\right)^{2 \alpha-2}}{\left(1-r_{0}\right)^{2 \alpha+2}}+(n-1) \frac{\left(1+r_{0}\right)^{2 \alpha-1}}{\left(1-r_{0}\right)^{2 \alpha+1}}
$$


Since the function

$$
\varphi(t, s)=\frac{(1+t)^{2 s-2}}{(1-t)^{2 s+2}}+(n-1) \frac{(1+t)^{2 s-1}}{(1-t)^{2 s+1}}, \quad 0<t<1
$$

is an increasing function of $s$, there is a number $\beta_{0}>\alpha$, such that

$$
\operatorname{Tr}\left({\overline{J_{F}\left(z_{0}\right)}}^{\prime} J_{F}\left(z_{0}\right)\right)=\varphi\left(r_{0}, \beta_{0}\right)
$$

Consequently, for small $\epsilon>0$ and $r_{0}-\epsilon<r<r_{0}+\epsilon$, there is a $C^{1}$ smooth function $\beta(r)$ such that $\beta\left(r_{0}\right)=\beta_{0}$ and

$$
\operatorname{Tr}\left({\overline{J_{F}\left(r u_{0}\right)}}^{\prime} J_{F}\left(r u_{0}\right)\right)=\frac{(1+r)^{2 \beta(r)-2}}{(1-r)^{2 \beta(r)+2}}+(n-1) \frac{(1+r)^{2 \beta(r)-1}}{(1-r)^{2 \beta(r)+1}}=\varphi(r, \beta(r))
$$

Thus

$$
\frac{\operatorname{Tr}\left({\overline{J_{F}\left(r u_{0}\right)}}^{\prime} J_{F}\left(r u_{0}\right)\right)-\operatorname{Tr}\left({\overline{J_{F}\left(z_{0}\right)}}^{\prime} J_{F}\left(z_{0}\right)\right)}{r-r_{0}}=\frac{\varphi(r, \beta(r))-\varphi\left(r_{0}, \beta_{0}\right)}{r-r_{0}} .
$$

By Lemma 2.5, we have

$$
\begin{aligned}
& \frac{\varphi(r, \beta(r))-\varphi\left(r_{0}, \beta_{0}\right)}{2\left(r-r_{0}\right)} \\
= & \frac{r_{0}}{1-r_{0}^{2}}\left[\operatorname{Tr}\left({\overline{J_{f}\left(z_{0}\right)}}^{\prime} J_{f}\left(z_{0}\right)\right)+\operatorname{Tr}\left[{\overline{u_{0}}}^{\prime}{\overline{J_{F}\left(z_{0}\right)}}^{\prime} J_{F}\left(z_{0}\right) u_{0}\right]\right] \\
+ & \frac{1}{1-r_{0}^{2}} \operatorname{Tr}\left(J_{F}\left(z_{0}\right){\overline{J_{F}\left(z_{0}\right)}}^{\prime} D^{2} F(0) u_{0}\right)+O\left(\left|r-r_{0}\right|\right) .
\end{aligned}
$$

As $r \rightarrow r_{0}$, the limit of the left hand side of $(2.15)$ is $\frac{1}{2}\left[\varphi_{r}+\beta^{\prime} \varphi_{\beta}\right]_{r=r_{0}}$. We evaluate it.

Noting that

$$
\begin{aligned}
\varphi_{r} & =\frac{\partial}{\partial r}\left[\frac{(1+r)^{2 \beta-2}}{(1-r)^{2 \beta+2}}+(n-1) \frac{(1+r)^{2 \beta-1}}{(1-r)^{2 \beta+1}}\right] \\
& =\frac{(1+r)^{2 \beta-2}}{(1-r)^{2 \beta+2}} \frac{4 \beta+4 r}{1-r^{2}}+(n-1) \frac{(1+r)^{2 \beta-1}}{(1-r)^{2 \beta+1}} \frac{4 \beta+2 r}{1-r^{2}} \\
& =\frac{4 \beta+4 r}{1-r^{2}}\left[\frac{(1+r)^{2 \beta-2}}{(1-r)^{2 \beta+2}}+(n-1) \frac{(1+r)^{2 \beta-1}}{(1-r)^{2 \beta+1}}\right]-\frac{2 r(n-1)}{1-r^{2}} \frac{(1+r)^{2 \beta-1}}{(1-r)^{2 \beta+1}}
\end{aligned}
$$

we have

$$
\left.\varphi_{r}\right|_{r=r_{0}}=\frac{4 \beta_{0}+4 r_{0}}{1-r_{0}^{2}} \operatorname{Tr}\left({\overline{J_{F}\left(z_{0}\right)}}^{\prime} J_{F}\left(z_{0}\right)\right)-\frac{2(n-1) r_{0}}{1-r_{0}^{2}} \frac{\left(1+r_{0}\right)^{2 \beta_{0}-1}}{\left(1-r_{0}\right)^{2 \beta_{0}+1}}
$$

by (2.13). 
Moreover,

$$
\begin{aligned}
\beta^{\prime} \varphi_{\beta} & =\beta^{\prime} \frac{\partial}{\partial \beta}\left[\frac{(1+r)^{2 \beta-2}}{(1-r)^{2 \beta+2}}+(n-1) \frac{(1+r)^{2 \beta-1}}{(1-r)^{2 \beta+1}}\right] \\
& =\frac{(1+r)^{2 \beta-2}}{(1-r)^{2 \beta+2}} 2 \beta^{\prime} \log \frac{1+r}{1-r}+\frac{(1+r)^{2 \beta-1}}{(1-r)^{2 \beta+1}} 2 \beta^{\prime} \log \frac{1+r}{1-r} \\
& =2 \beta^{\prime} \log _{\frac{1+r}{1-r}}\left[\frac{(1+r)^{2 \beta-2}}{(1-r)^{2 \beta+2}}+(n-1) \frac{(1+r)^{2 \beta-1}}{(1-r)^{2 \beta+1}}\right]
\end{aligned}
$$

we have

$$
\left.\beta^{\prime} \varphi_{\beta}\right|_{r=r_{0}}=2 \beta^{\prime}\left(r_{0}\right) \log \frac{1+r_{0}}{1-r_{0}} \operatorname{Tr}\left({\overline{J_{F}\left(z_{0}\right)}}^{\prime} J_{F}\left(z_{0}\right)\right)
$$

by $(2.13)$.

Thus

$$
\begin{aligned}
& \frac{1}{2}\left[\varphi_{r}+\beta^{\prime} \varphi_{\beta}\right]_{r=r_{0}} \\
= & \left(\frac{2 \beta_{0}+2 r_{0}}{1-r_{0}^{2}}+\beta^{\prime}\left(r_{0}\right) \log _{\frac{1+r_{0}}{1-r_{0}}}\right) \operatorname{Tr}\left(\overline{J_{F}\left(z_{0}\right)^{\prime}} J_{F}\left(z_{0}\right)\right) \\
& -\frac{(n-1) r_{0}}{1-r_{0}^{2}} \frac{\left(1+r_{0}\right)^{2 \beta_{0}-1}}{\left(1-r_{0}\right)^{2 \beta_{0}+1}} .
\end{aligned}
$$

As $r \rightarrow r_{0},(2.15)$ becomes the following equality

$$
\begin{aligned}
& \left(\frac{2 \beta_{0}+2 r_{0}}{1-r_{0}^{2}}+\beta^{\prime}\left(r_{0}\right) \log \frac{1+r_{0}}{1-r_{0}}\right) \operatorname{Tr}\left({\overline{J_{F}\left(z_{0}\right)}}^{\prime} J_{F}\left(z_{0}\right)\right)-\frac{(n-1) r_{0}}{1-r_{0}^{2}} \frac{\left(1+r_{0}\right)^{2 \beta_{0}-1}}{\left(1-r_{0}\right)^{2 \beta_{0}+1}} \\
= & \frac{r_{0}}{1-r_{0}^{2}}\left[\operatorname{Tr}\left({\overline{J_{F}\left(z_{0}\right)}}^{\prime} J_{F}\left(z_{0}\right)\right)+\operatorname{Tr}\left({\overline{u_{0}}}^{\prime}{\overline{J_{F}\left(z_{0}\right)}}^{\prime} J_{F}\left(z_{0}\right) u_{0}\right)\right] \\
& +\frac{1}{1-r_{0}^{2}} \operatorname{Tr}\left(J_{F}\left(z_{0}\right){\overline{J_{F}\left(z_{0}\right)}}^{\prime} D^{2} F(0) u_{0}\right)
\end{aligned}
$$

by (2.16). After cancel the term $\frac{r_{0}}{1-r_{0}^{2}} \operatorname{Tr}\left(\overline{J_{F}\left(z_{0}\right)} J_{F}\left(z_{0}\right)\right)$ on both sides of the privious equality, we have

$$
\begin{aligned}
& \left(\frac{2 \beta_{0}+r_{0}}{1-r_{0}^{2}}+\beta^{\prime}\left(r_{0}\right) \log \frac{1+r_{0}}{1-r_{0}}\right) \operatorname{Tr}\left(\overline{J_{F}\left(z_{0}\right)} J_{F}\left(z_{0}\right)\right)-\frac{(n-1) r_{0}}{1-r_{0}^{2}} \frac{\left(1+r_{0}\right)^{2 \beta_{0}-1}}{\left(1-r_{0}\right)^{2 \beta_{0}+1}} \\
= & \frac{r_{0}}{1-r_{0}^{2}}{\overline{u_{0}}}^{\prime}{\overline{J_{F}\left(z_{0}\right)}}^{\prime} J_{F}\left(z_{0}\right) u_{0}+\frac{1}{1-r_{0}^{2}} \operatorname{Tr}\left(J_{F}\left(z_{0}\right){\overline{J_{F}\left(z_{0}\right)}}^{\prime} D^{2} F(0) u_{0}\right) .
\end{aligned}
$$

By equality (2.17), we know that $\operatorname{Tr}\left(J_{F}\left(z_{0}\right){\overline{J_{F}\left(z_{0}\right)}}^{\prime} D^{2} F(0) u_{0}\right)$ is real. $J_{F}\left(z_{0}\right){\overline{J_{F}\left(z_{0}\right.}}^{\prime}$ is a positive definite Hermitian matrix, we may decompose it as

$$
J_{F}\left(z_{0}\right){\overline{J_{F}\left(z_{0}\right)}}^{\prime}=U\left(\begin{array}{ccc}
\lambda_{1}^{2} & & \\
& \ddots & \\
& & \lambda_{n}^{2}
\end{array}\right) \bar{U}^{\prime},
$$


where $U$ is an unitary matrix. Thus

$$
\begin{aligned}
& \operatorname{Tr}\left(J_{F}\left(z_{0}\right) \overline{J_{F}\left(z_{0}\right)}{ }^{\prime} D^{2} F(0) u_{0}\right) \\
= & \operatorname{Tr}\left(U\left(\begin{array}{ccc}
\lambda_{1}^{2} & & \\
& \ddots & \\
& & \lambda_{n}^{2}
\end{array}\right) \bar{U}^{\prime} D^{2} F(0) u_{0}\right) \\
= & \operatorname{Tr}\left(\left(\begin{array}{ccc}
\lambda_{1}^{2} & & \\
& \ddots & \\
& & \lambda_{n}^{2}
\end{array}\right) \bar{U}^{\prime} D^{2} F(0) u_{0} U\right) .
\end{aligned}
$$

If

$$
\bar{U}^{\prime} D^{2} F(0) u_{0} U=\left(\begin{array}{ccc}
a_{11} & \cdots & a_{1 n} \\
\cdots & \cdots & \cdots \\
a_{n 1} & \cdots & a_{n n}
\end{array}\right)
$$

then

$$
\left|\operatorname{Tr}\left(\left(\begin{array}{ccc}
\lambda_{1}^{2} & & \\
& \ddots & \\
& & \lambda_{n}^{2}
\end{array}\right) \bar{U}^{\prime} D^{2} F(0) u_{0} U\right)\right|=\left|\sum_{j=1}^{n} \lambda_{j}^{2} a_{j j}\right| \leq \sum_{j=1}^{n} \lambda_{j}^{2}\left|a_{j j}\right|
$$

But

$$
\left|a_{j j}\right| \leq\left\|\bar{U}^{\prime} D^{2} F(0) u_{0} U\right\|=\left\|D^{2} F(0) u_{0}\right\| \leq 2 \alpha, j=1,2,3, \cdots \cdots, n,
$$

since the $\|$ ord $\| \mathcal{S}=\alpha$ and (1.20). Thus

$$
\left|\operatorname{Tr}\left(J_{F}\left(z_{0}\right){\overline{J_{F}\left(z_{0}\right)}}^{\prime} D^{2} F(0) u_{0}\right)\right| \leq 2 \alpha \operatorname{Tr}\left({\overline{J_{F}\left(z_{0}\right)}}^{\prime} J_{F}\left(z_{0}\right)\right) .
$$

By (1.21)(Theorem 4.1 in [14]), and $\beta_{0}>\alpha$, we have

$$
\begin{aligned}
{\overline{u_{0}}}^{\prime}{\overline{J_{F}\left(z_{0}\right)}}^{\prime} J_{F}\left(z_{0}\right) u_{0} & \leq \text { The largest eigenvalue of } \overline{J_{F}\left(z_{0}\right)} J_{F}\left(z_{0}\right) \\
& \leq \frac{\left(1+r_{0}\right)^{2 \alpha-2}}{\left(1-r_{0}\right)^{2 \alpha+2}} \\
& <\frac{\left(1+r_{0}\right)^{2 \beta_{0}-2}}{\left(1-r_{0}\right)^{2 \beta_{0}+2}}
\end{aligned}
$$

From (2.17), (2.18) and (2.19), we obtain the following inequality (2.20)

$$
\begin{aligned}
& \left(\frac{2 \beta_{0}+r_{0}}{1-r_{0}^{2}}+\beta^{\prime}\left(r_{0}\right) \log \frac{1+r_{0}}{1-r_{0}}\right) \operatorname{Tr}\left({\overline{J_{F}\left(z_{0}\right)}}^{\prime} J_{F}\left(z_{0}\right)\right) \\
\leq & \frac{\left(1+r_{0}\right)^{2 \beta_{0}-2}}{\left(1-r_{0}\right)^{2 \beta_{0}+2}} \frac{r_{0}}{1-r_{0}^{2}}+\frac{(n-1) r_{0}}{1-r_{0}^{2}} \frac{\left(1+r_{0}\right)^{2 \beta_{0}-1}}{\left(1-r_{0}\right)^{2 \beta_{0}+1}}+\frac{2 \alpha}{1-r_{0}^{2}} \operatorname{Tr}\left(\overline{J_{F}\left(z_{0}\right)} J_{F}\left(z_{0}\right)\right) \\
= & \frac{r_{0}}{1-r_{0}^{2}} \operatorname{Tr}\left({\overline{J_{F}\left(z_{0}\right)}}^{\prime} J_{F}\left(z_{0}\right)\right)+\frac{2 \alpha}{1-r_{0}^{2}} \operatorname{Tr}\left({\overline{J_{F}\left(z_{0}\right)}}^{\prime} J_{F}\left(z_{0}\right)\right)
\end{aligned}
$$


by (2.13). After cancel the term $\frac{r_{0}}{1-r_{0}^{2}} \operatorname{Tr}\left({\overline{J_{F}\left(z_{0}\right)}}^{\prime} J_{F}\left(z_{0}\right)\right)$ on both sides of (2.20), we have

$$
\left(\frac{2 \beta_{0}}{1-r_{0}^{2}}+\beta^{\prime}\left(r_{0}\right) \log \frac{1+r_{0}}{1-r_{0}}\right) \operatorname{Tr}\left({\overline{J_{F}\left(z_{0}\right)}}^{\prime} J_{F}\left(z_{0}\right)\right) \leq \frac{2 \alpha}{1-r_{0}^{2}} \operatorname{Tr}\left(\overline{J_{F}\left(z_{0}\right)} J_{F}^{\prime}\left(z_{0}\right)\right) \text {. }
$$

From (2.21), we conclude $\beta^{\prime}\left(r_{0}\right)<0$ since $\beta_{0}>\alpha$. Hence there is $r_{1}<r_{0}$ such that $\beta_{1}=\beta\left(r_{1}\right)>\beta\left(r_{0}\right)=\beta_{0}>\alpha$, and

$$
\begin{aligned}
\operatorname{Tr}\left({\left.\overline{J_{F}\left(r_{1} u_{0}\right)^{\prime}} J_{F}\left(r_{1} u_{0}\right)\right)}=\frac{\left(1+r_{1}\right)^{2 \beta_{1}-2}}{\left(1-r_{1}\right)^{2 \beta_{1}+2}}+(n-1) \frac{\left(1+r_{1}\right)^{2 \beta_{1}-1}}{\left(1-r_{1}\right)^{2 \beta_{1}+1}}\right. \\
>\frac{\left(1+r_{1}\right)^{2 \beta_{0}-2}}{\left(1-r_{1}\right)^{2 \beta_{0}+2}}+(n-1) \frac{\left(1+r_{1}\right)^{2 \beta_{0}-1}}{\left(1-r_{1}\right)^{2 \beta_{0}+1}}
\end{aligned}
$$

by $(2.14)$

Let $u_{1} \in \partial \boldsymbol{B}^{n}$, such that

$$
\operatorname{Tr}\left(\overline{J_{F}^{\left(r_{1} u_{1}\right)}} J_{F}\left(r_{1} u_{1}\right)\right)=\max _{u \in \partial \boldsymbol{B}^{n}} \operatorname{Tr}\left({\overline{J_{F}\left(r_{1} u\right)}}^{\prime} J_{F}\left(r_{1} u\right)\right)
$$

Write $z_{1}=r_{1} u_{1}$, let $F_{1}$ be an extremal such that

$$
\operatorname{Tr}\left({\overline{J_{F_{1}}\left(z_{1}\right)}}^{\prime} J_{F_{1}}\left(z_{1}\right)\right)=\max _{f \in \mathcal{S}} \operatorname{Tr}\left({\overline{J_{f}\left(z_{1}\right)}}^{\prime} J_{f}\left(z_{1}\right)\right)
$$

By (2.22), (2.23), we have

$$
\begin{aligned}
\operatorname{Tr}\left({\overline{J_{F_{1}}\left(z_{1}\right)}}^{\prime} J_{F_{1}}\left(z_{1}\right)\right) & \geq \frac{\left(1+r_{1}\right)^{2 \beta_{1}-2}}{\left(1-r_{1}\right)^{2 \beta_{1}+2}}+(n-1) \frac{\left(1+r_{1}\right)^{2 \beta_{1}-1}}{\left(1-r_{1}\right)^{2 \beta_{1}+1}} \\
& >\frac{\left(1+r_{1}\right)^{2 \beta_{0}-2}}{\left(1-r_{1}\right)^{2 \beta_{0}+2}}+(n-1) \frac{\left(1+r_{1}\right)^{2 \beta_{0}-1}}{\left(1-r_{1}\right)^{2 \beta_{0}+1}}
\end{aligned}
$$

We can repeat the argument just given. Thus one proves that there are a sequence of numbers $\left\{r_{i}\right\}$ decreasing to zero and a sequence $\left\{F_{j}\right\} \subset \mathcal{S}$, such that

$$
\begin{aligned}
& \operatorname{Tr}\left(\overline{J_{F_{1}}\left(z_{1}\right)} J_{F_{1}}\left(z_{1}\right)\right) \\
> & \frac{\left(1+r_{j}\right)^{2 \beta_{0}-2}}{\left(1-r_{j}\right)^{2 \beta_{0}+2}}+(n-1) \frac{\left(1+r_{j}\right)^{2 \beta_{0}-1}}{\left(1-r_{j}\right)^{2 \beta_{0}+1}} \\
= & \left(1+\left(2 \beta_{0}-2\right) r_{j}+\cdots\right)\left(1-\left(2 \beta_{0}+2\right) r_{j}+\cdots\right)^{-1} \\
+ & (n-1)\left(1+\left(2 \beta_{0}-2\right) r_{j}+\cdots\right)\left(1-\left(2 \beta_{0}+2\right) r_{j}+\cdots\right)^{-1} \\
= & n+4 \beta_{0} n r_{j}+O\left(r_{j}^{2}\right),
\end{aligned}
$$

where $z_{j}=r_{j} u_{j}, u_{j} \in \partial \boldsymbol{B}^{n}, j=1,2, \cdots \cdots$.

Since $J_{F_{j}}\left(z_{j}\right)=I+D^{2} F_{j}(0) z_{j}+O\left(\left|z_{j}\right|^{2}\right)$, we have

$$
{\overline{J_{F_{j}}\left(z_{j}\right)}}^{\prime} J_{F_{j}}\left(z_{j}\right)=I+D^{2} F_{j}(0) z_{j}+{\overline{D^{2} F_{j}(0) z_{j}}}^{\prime}+O\left(\left|z_{j}\right|^{2}\right),
$$




$$
D^{2} F_{j}(0) z_{j}+{\overline{D^{2} F_{j}(0) z_{j}}}^{\prime}+O\left(\left|z_{j}\right|^{2}\right)={\overline{J_{F_{j}}\left(z_{j}\right)}}^{\prime} J_{F_{j}}\left(z_{j}\right)-I
$$

and hence

(2.25)

$\operatorname{Tr}\left(D^{2} F_{j}(0) z_{j}+{\overline{D^{2} F_{j}(0) z_{j}}}^{\prime}\right)+O\left(\left|z_{j}\right|^{2}\right)=\operatorname{Tr}\left({\overline{J_{F_{j}}\left(z_{j}\right)}}^{\prime} J_{F_{j}}\left(z_{j}\right)\right)-n>4 \beta_{0} n r_{j}+O\left(\left|z_{j}\right|^{2}\right)$

by $(2.24)$

Recall that $\mathcal{S}$ is a normal family when $\|$ ord $\| \mathcal{S}=\alpha<\infty$, there is a subsequence of $\left\{F_{j}\right\}$ that converges locally uniformly to a mapping $f \in \mathcal{S}$ and $u_{j}$ tend to a unit vector $u$. Thus dividing by $r_{j}$ on both sides of (2.25) and letting $j \rightarrow \infty$ through the subsequence, we obtain

$$
\operatorname{Tr}\left(D^{2} f(0) u+{\overline{D^{2} f(0) u}}^{\prime}\right) \geq 4 \beta_{0} n .
$$

But

$$
\operatorname{Tr}\left(D^{2} f(0) u\right)+\operatorname{Tr}\left({\overline{D^{2} f(0) u}}^{\prime}\right) \leq 2\left|\operatorname{Tr} D^{2} f(0) u\right| \leq 2 n\left\|D^{2} f(0) u\right\|
$$

we have

$$
2 n\left\|D^{2} f(0) u\right\| \geq 4 \beta_{0} n>4 \alpha n
$$

i.e.,

$$
\left\|D^{2} f(0) u\right\|>2 \alpha=2\|\operatorname{ord}\| \mathcal{S} .
$$

by (2.26). This contradiction completes the proof of the upper bound inequality in Theorem 2.1.

The proof of the lower bound inequality in Theorem 2.1 is similar with the proof of upper bound inequality.

Throughout this argument, $F$ will be an extremal mapping for the minimum problem associated with a fixed point $z_{0} \in \boldsymbol{B}^{n}$, i.e.,

$$
\operatorname{Tr}\left({\overline{J_{F}\left(z_{0}\right)}}^{\prime} J_{F}\left(z_{0}\right)\right) \leq \operatorname{Tr}\left(\overline{J_{f}\left(z_{0}\right)} J_{f}\left(z_{0}\right)\right)
$$

holds for all $f \in \mathcal{S}$. We may prove the Lemma 2.2, 2.3, 2.4 and 2.5 under the assumption (2.27), and prove the lower bound of the inequality if we instead $\varphi(t, s)$ by

$$
\eta(t, s)=\varphi(-t, s)=\frac{(1-t)^{2 s-2}}{(1+t)^{2 s+2}}+(n-1) \frac{(1-t)^{2 s-1}}{(1+t)^{2 s+1}} .
$$

All the argument of the proof are similar with the proof of the upper bound. We omit the detail.

3. The estimations of the Jacobian of mappings in a $\boldsymbol{B}^{n}$. We may use the method as we used in the previous section to estimate $\operatorname{det}\left(J_{f}(z){\overline{J_{f}(z)}}^{\prime}\right)$ of mapping $f$ in a LIF on the unit ball $\boldsymbol{B}^{n}$ in $\mathbb{C}^{n}$. Actually, the following theorem about the estimations of $\operatorname{det}\left(J_{f}(z){\overline{J_{f}(z)}}^{\prime}\right)$ was known long time ago, which was proved by Barnard,FifzGerald and Gong [1], Liu [11], Gong and Zheng [8], and Pfaltzgraff [12]. In this section, the proof of Theorm 3.1 is different with the original proof. It also shows that using $C(\mathcal{S})$ 
which was defined by (1.5) and (1.19) to estimate $\operatorname{det}\left(J_{f}(z){\overline{J_{f}(z)}}^{\prime}\right)$ is the right choice. In this section, all the vectors are column vectors.

THEOREM 3.1. If $\mathcal{S}$ is a LIF on the unit ball $\boldsymbol{B}^{n}$ in $\mathbb{C}^{n}$, then

$$
\frac{(1-|z|)^{2 C(\mathcal{S})-(n+1)}}{(1+|z|)^{2 C(\mathcal{S})+(n+1)}} \leq \operatorname{det}\left({\overline{J_{f}(z)}}^{\prime} J_{f}(z)\right) \leq \frac{(1+|z|)^{2 C(\mathcal{S})-(n+1)}}{(1-|z|)^{2 C(\mathcal{S})+(n+1)}}
$$

for all $f \in \mathcal{S}$, and $z \in \boldsymbol{B}^{n}$, where $C(\mathcal{S})$ is defined by (1.5) and (1.19).

First of all, we prove the following Lemmas.

LeMmA 3.2. Fix $z_{0}=r_{0} u_{0} \in \boldsymbol{B}^{n}, u_{0} \in \partial \boldsymbol{B}^{n}$. If $F$ is the extremal mapping in $\mathcal{S}$ for the following maximum problem:

$$
\operatorname{det}\left({\overline{J_{F}\left(z_{0}\right)}}^{\prime} J_{F}\left(z_{0}\right)\right) \geq \operatorname{det}\left({\overline{J_{f}\left(z_{0}\right)}}^{\prime} J_{f}\left(z_{0}\right)\right)
$$

holds for any $f \in \mathcal{S}$, then

$$
\operatorname{det}\left(J_{F}\left(z_{0}\right){\overline{J_{F}\left(z_{0}\right)}}^{\prime}\right)=\max \left\{\operatorname{det}\left(J_{F}(z){\overline{J_{F}(z)}}^{\prime}\right)|| z \mid=r_{0}\right\} .
$$

Proof. If (3.2) were false, then there be a point $z_{1} \neq z_{0},\left|z_{1}\right|=r_{0}$ such that

$$
\operatorname{det}\left(\overline{J_{F}\left(z_{1}\right)} J^{\prime} J_{F}\left(z_{1}\right)\right)>\operatorname{det}\left(\overline{J_{F}\left(z_{0}\right)} J_{F}\left(z_{0}\right)\right) .
$$

There exists an unitary matrix $U$ such that $U z_{0}=z_{1}$ where $z_{0}, z_{1}$ are column vectors. Consider $F_{U}(z)=U^{-1} F(U z) \in \mathcal{S}$, then $J_{F_{U}}(z)=U^{-1} J_{F}(U z) U$, and consequently

$$
\operatorname{det}\left({\overline{J_{F_{U}}(z)}}^{\prime} J_{F_{U}}(z)\right)=\operatorname{det}\left({\overline{J_{F}(U z)}}^{\prime} J_{F}(U z)\right)
$$

which yied the contradiction

$$
\operatorname{det}\left({\overline{J_{F_{U}}\left(z_{0}\right)}}^{\prime} J_{F_{U}}\left(z_{0}\right)\right)=\operatorname{det}\left({\overline{J_{F}\left(z_{1}\right)}}^{\prime} J_{F}\left(z_{1}\right)\right)>\operatorname{det}\left({\overline{J_{F}\left(z_{0}\right)}}^{\prime} J_{F}\left(z_{0}\right)\right) .
$$

Lemma 3.3. Fix $z_{0}=r_{0} u_{0} \in \boldsymbol{B}^{n}, u_{0} \in \partial \boldsymbol{B}^{n}$. If $F$ is the extremal mapping in $\mathcal{S}$ for the maximum problem in Lemma 3.2, then $\operatorname{Tr}\left(D^{2} F\left(z_{0}\right) z_{0}\left(J_{F}\left(z_{0}\right)\right)^{-1}\right)$ is real, equivalently, $\operatorname{Tr}\left(D^{2} F\left(z_{0}\right) u_{0}\left(J_{F}\left(z_{0}\right)\right)^{-1}\right)$ is real.

Proof. Let $t$ be a small real number, then by (2.5),

$$
\begin{aligned}
& \operatorname{det}\left({\overline{J_{F}\left(z_{0} e^{i t}\right)}}^{\prime} J_{F}\left(z_{0} e^{i t}\right)\right) \\
& =\operatorname{det}\left[\overline { J _ { F } ( z _ { 0 } ) } \left(I+i t D^{2} F\left(z_{0}\right) z_{0}\left(J_{F}\left(z_{0}\right)\right)^{-1}\right.\right. \\
& \left.\left.-i t\left(\overline{J_{F}\left(z_{0}\right)}\right)^{\prime}{\overline{\left(D^{2} F\left(z_{0}\right) z_{0}\right)}}^{\prime}+O\left(t^{2}\right)\right) J_{F}\left(z_{0}\right)\right] \\
& =\operatorname{det}\left({\overline{J_{F}\left(z_{0}\right)}}^{\prime} J_{F}\left(z_{0}\right)\right)\left[1+t \operatorname{Tr}\left[i t D^{2} F\left(z_{0}\right) z_{0}\left(J_{F}\left(z_{0}\right)\right)^{-1}-i{\overline{\left(J_{F}\left(z_{0}\right)\right)^{-1}}}^{\prime} \overline{\left(D^{2} F\left(z_{0}\right) z_{0}\right)}\right]\right. \\
& +O\left(t^{2}\right)
\end{aligned}
$$

since

$$
\operatorname{det}(I+t H)=1+t \operatorname{Tr} H+O\left(t^{2}\right)
$$


when $t$ is a small real number, $H$ is a Hermitian matrix. By Lemma 3.2,

$$
\begin{aligned}
0 & \geq \operatorname{det}\left(\overline{J_{F}\left(z_{0} e^{i t}\right)}{ }^{\prime} J_{F}\left(z_{0} e^{i t}\right)\right)-\operatorname{det}\left(\overline{J_{F}\left(z_{0}\right)} J_{F}\left(z_{0}\right)\right) \\
= & \operatorname{det}\left({\overline{J_{F}\left(z_{0}\right)}}^{\prime} J_{F}\left(z_{0}\right)\right) t \operatorname{tTr}\left[i \left(D^{2} F\left(z_{0}\right) z_{0}\left(J_{F}\left(z_{0}\right)\right)^{-1}\right.\right. \\
& \left.\left.-\left(\overline{J_{F}\left(z_{0}\right)^{\prime}}\right)^{-1}\left(\overline{D^{2} F\left(z_{0}\right) z_{0}}\right)^{\prime}\right)\right]+O\left(t^{2}\right) .
\end{aligned}
$$

Dividing by $|t|$ and considering both $t \rightarrow 0^{+}$and $t \rightarrow 0^{-}$, we have

$$
\operatorname{Im}\left\{\operatorname{Tr}\left(D^{2} F\left(z_{0}\right) z_{0}\left(J_{F}\left(z_{0}\right)\right)^{-1}\right\}=0 .\right.
$$

LeMma 3.4. Fix $z_{0}=r_{0} u_{0} \in \boldsymbol{B}^{n}, u_{0} \in \partial \boldsymbol{B}^{n}$. If $F$ is the extremal mapping in $\mathcal{S}$ for the maximum problem in Lemma 3.2, then

$$
\begin{aligned}
\operatorname{Tr}\left(D^{2} F\left(z_{0}\right) \nu\left(J_{F}\left(z_{0}\right)\right)^{-1}\right)-\bar{z}^{\prime} \nu \operatorname{Tr}\left(D^{2} F\left(z_{0}\right) z_{0}\left(J_{F}\left(z_{0}\right)\right)^{-1}\right) \\
-(n+1){\overline{z_{0}}}^{\prime} \nu-\operatorname{Tr}\left(D^{2} F(0) \nu\right)=0
\end{aligned}
$$

holds for any $\nu \in \partial \boldsymbol{B}^{n}$, where $z_{0}, \nu$ are column vectors.

Proof. From (2.7), we have

$$
\begin{aligned}
& \operatorname{det}\left(\overline{J_{F}(z ; a)} J_{F}(z ; a)\right) \\
= & \operatorname{det}\left({\overline{J_{F}(z)}}^{\prime} J_{F}(z)\right) \operatorname{det}\left[I+2 \Re e\left(\left(D^{2} F(z)\left(a-\bar{a}^{\prime} z z\right)-J_{F}(z) z \bar{a}^{\prime}\right.\right.\right. \\
- & \left.\left.\left.\bar{a}^{\prime} z J_{F}(z)-D^{2} F(0) a J_{F}(z)\right)\left(J_{F}(z)\right)^{-1}\right)+O\left(|a|^{2}\right)\right] \\
= & \operatorname{det}\left({\overline{J_{F}(z)}}^{\prime} J_{F}(z)\right)\left(1+2 \Re e\left[\operatorname { T r } \left(D^{2} F(z)\left(a-\bar{a}^{\prime} z z\right)\left(J_{F}(z)\right)^{-1}\right.\right.\right. \\
- & \left.\left.(n+1) \bar{a}^{\prime} z-\operatorname{Tr}\left(D^{2} F(0) a\right)\right]+O\left(|a|^{2}\right)\right)
\end{aligned}
$$

when $|a|$ is small. By the extremal property of $F$ at $z_{0}$, we have

$$
\begin{aligned}
0 & \geq \operatorname{det}\left({\overline{J_{F}(z ; a)}}^{\prime} J_{F}(z ; a)\right)-\operatorname{det}\left({\overline{J_{F}\left(z_{0}\right)}}^{\prime} J_{F}\left(z_{0}\right)\right) \\
& =\operatorname{det}\left({\overline{J_{F}\left(z_{0}\right)}}^{\prime} J_{F}\left(z_{0}\right)\right) \cdot 2 \Re e\left[\operatorname{Tr}\left(D^{2} F\left(z_{0}\right)\left(a-\bar{a}^{\prime} z_{0} z_{0}\right)\left(J_{F}\left(z_{0}\right)\right)^{-1}\right)\right. \\
& \left.-(n+1) \bar{a}^{\prime} z_{0}-\operatorname{Tr}\left(D^{2} F(0) a\right)\right]+O\left(|a|^{2}\right) .
\end{aligned}
$$

By Lemma 3.3, we know that $\operatorname{Tr}\left(D^{2} F\left(z_{0}\right) z_{0}\left(J_{F}\left(z_{0}\right)\right)^{-1}\right)$ is real, thus

$$
\Re e\left[\operatorname{Tr}\left(D^{2} F\left(z_{0}\right) \bar{a}^{\prime} z_{0} z_{0}\left(J_{F}\left(z_{0}\right)\right)^{-1}\right)\right]=\Re e\left[\operatorname{Tr}\left(D^{2} F\left(z_{0}\right){\overline{z_{0}}}^{\prime} a z_{0}\left(J_{F}\left(z_{0}\right)\right)^{-1}\right)\right] .
$$

Substituting it into previous inequality, we have

$$
\begin{aligned}
0 & \geq \operatorname{det}\left(\overline{J_{F}\left(z_{0}\right)} J_{F}\left(z_{0}\right)\right) \Re e\left[\operatorname{Tr}\left(D^{2} F\left(z_{0}\right)\left(a-\bar{a}^{\prime} z_{0} z_{0}\right)\left(J_{F}\left(z_{0}\right)\right)^{-1}\right)\right. \\
& \left.-(n+1) \bar{a}^{\prime} z_{0}-\operatorname{Tr}\left(D^{2} F(0) a\right)\right]+O\left(|a|^{2}\right) .
\end{aligned}
$$

Dividing $|a|$ on both sides of the previous inequality, and denoting $\nu=\frac{a}{|a|}$, then let $|a| \rightarrow 0$, note that $a$ can be replaced by $e^{i t} a$ with arbitrary real $t$, we have proved (3.3). 
LemMA 3.5. Fix $z_{0}=r_{0} u_{0} \in \boldsymbol{B}^{n}, u_{0} \in \partial \boldsymbol{B}^{n}$. If $F$ is the extremal mapping in $\mathcal{S}$ for the maximum problem in Lemma 3.2, then

$$
\begin{aligned}
& \frac{\operatorname{det}\left({\overline{J_{F}(z)}}^{\prime} J_{F}(z)\right)-\operatorname{det}\left(\overline{J_{F}\left(z_{0}\right)}{ }^{\prime} J_{F}\left(z_{0}\right)\right)}{2\left(r-r_{0}\right)} \\
= & \operatorname{det}\left({\overline{J_{F}\left(z_{0}\right)}}^{\prime} J_{F}\left(z_{0}\right)\right) \frac{(n+1) r_{0}+T r\left(D^{2} F(0) u_{o}\right)}{1-r_{0}^{2}}+O\left(\left|r-r_{0}\right|\right)
\end{aligned}
$$

where $z=r u_{0}$, and $r$ is a real number near $r_{0}$.

Proof. Expanding $J_{F}(z)$ at $z_{0}$, we have

$$
\begin{aligned}
& \operatorname{det}\left(\overline{J_{F}(z)}{ }^{\prime} J_{F}(z)\right) \\
= & \operatorname{det}\left({\overline{J_{F}\left(z_{0}\right)}}^{\prime} J_{F}\left(z_{0}\right)\right)\left(1+2 \Re e\left[\operatorname{Tr}\left(D^{2} F\left(z_{0}\right)\left(z-z_{0}\right)\left(J_{F}\left(z_{0}\right)\right)^{-1}\right)\right]+O\left(\left|z-z_{0}\right|^{2}\right)\right.
\end{aligned}
$$

by (2.9). Dividing $2\left(r-r_{0}\right)$ on both sides, it is

$$
\begin{aligned}
& \frac{\operatorname{det}\left({\overline{J_{F}(z)}}^{\prime} J_{F}(z)\right)-\operatorname{det}\left(\overline{J_{F}\left(z_{0}\right)} J_{F}\left(z_{0}\right)\right)}{2\left(r-r_{0}\right)} \\
= & \operatorname{det}\left({\overline{J_{F}\left(z_{0}\right)}}^{\prime} J_{F}\left(z_{0}\right)\right) \Re e\left[\operatorname{Tr}\left(D^{2} F\left(z_{0}\right) u_{0}\left(J_{F}\left(z_{0}\right)\right)^{-1}\right)\right]+O\left(\left|r-r_{0}\right|\right) .
\end{aligned}
$$

By Lemma 3.3, we know that

$$
\Re e\left[\operatorname{Tr}\left(D^{2} F\left(z_{0}\right) u_{0}\left(J_{F}\left(z_{0}\right)\right)^{-1}\right]=\operatorname{Tr}\left(D^{2} F\left(z_{0}\right) u_{0}\left(J_{F}\left(z_{0}\right)\right)^{-1}\right) .\right.
$$

Thus

$$
\begin{aligned}
& \frac{\operatorname{det}\left(\overline{J_{F}(z)}{ }^{\prime} J_{F}(z)\right)-\operatorname{det}\left({\overline{J_{F}\left(z_{0}\right)}}^{\prime} J_{F}\left(z_{0}\right)\right)}{2\left(r-r_{0}\right)} \\
= & \operatorname{det}\left({\overline{J_{F}\left(z_{0}\right.}}^{\prime} J_{F}\left(z_{0}\right)\right)\left[\operatorname{Tr}\left(D^{2} F\left(z_{0}\right) u_{0}\left(J_{F}\left(z_{0}\right)\right)^{-1}\right)\right]+O\left(\left|r-r_{0}\right|\right) .
\end{aligned}
$$

Let $\nu=u_{0}$ in Lemma 3.4, (3.3) becomes

$$
\begin{aligned}
& \operatorname{Tr}\left(D^{2} F\left(z_{0}\right) u_{0}\left(J_{F}\left(z_{0}\right)\right)^{-1}\right) \\
= & {\overline{z_{0}}}^{\prime} u_{0} \operatorname{Tr}\left(D^{2} F\left(z_{0}\right) z_{0}\left(J_{F}\left(z_{0}\right)\right)^{-1}\right)+(n+1){\overline{z_{0}}}^{\prime} u_{0}+\operatorname{Tr}\left(D^{2} F(0) u_{0}\right) \\
= & {r_{0}^{2}}^{2} \operatorname{Tr}\left(D^{2} F\left(z_{0}\right) u_{0}\left(J_{F}\left(z_{0}\right)\right)^{-1}\right)+(n+1) r_{0}+\operatorname{Tr}\left(D^{2} F(0) u_{0}\right) .
\end{aligned}
$$

Hence

$$
\operatorname{Tr}\left(D^{2} F\left(z_{0}\right) u_{0}\left(J_{F}\left(z_{0}\right)\right)^{-1}\right)=\frac{(n+1) r_{0}+\operatorname{Tr}\left(D^{2} F(0) u_{0}\right)}{1-r_{0}^{2}}
$$

Substituting (3.6) into (3.5), we get (3.4).

Proof of Theorem 3.1. The upper bound: Let $F$ be the extremal mapping for the maximum probrem in Lemma 3.2. We assume that the upper bound in (3.1) is false. Hence there exists $r_{0} \in(0,1), z_{0} \in \boldsymbol{B}^{n},\left|z_{0}\right|=r_{0}$ such that

$$
\operatorname{det}\left({\overline{J_{F}\left(z_{0}\right)}}^{\prime} J_{F}\left(z_{0}\right)\right)>\frac{\left(1+r_{0}\right)^{2 C(\mathcal{S})-(n+1)}}{\left(1-r_{0}\right)^{2 C(\mathcal{S})+(n+1)}}
$$


Since the function

$$
\zeta(t, s)=\frac{(1+t)^{2 s-(n+1)}}{(1-t)^{2 s+(n+1)}}, \quad 0<t<1,
$$

is an increasing function of $s$, there is a number $\beta_{0}>C(\mathcal{S})$ such that

$$
\operatorname{det}\left(\overline{J_{F}\left(z_{0}\right)} J_{F}\left(z_{0}\right)\right)=\zeta\left(r_{0}, \beta_{0}\right) .
$$

Consequently, for small $\epsilon>0$ and $r_{0}-\epsilon<r<r_{0}+\epsilon$, there is a $C^{1}$ smooth function $\beta(r)$ such that $\beta\left(r_{0}\right)=\beta_{0}$ and

$$
\operatorname{det}\left(\overline{J_{F}\left(r u_{0}\right)} J_{F}^{\prime}\left(r u_{0}\right)\right)=\frac{(1+r)^{2 \beta(r)-(n+1)}}{(1-r)^{2 \beta(r)+(n+1)}}=\zeta(r, \beta(r)) .
$$

Thus

$$
\frac{\operatorname{det}\left({\overline{J_{F}\left(r u_{0}\right)}}^{\prime} J_{F}\left(r u_{0}\right)\right)-\operatorname{det}\left({\overline{J_{F}\left(z_{0}\right)}}^{\prime} J_{F}\left(z_{0}\right)\right)}{r-r_{0}}=\frac{\zeta(r, \beta(r))-\zeta\left(r_{0}, \beta_{0}\right)}{r-r_{0}} .
$$

By Lemma 3.5, we have

(3.10)

$\frac{\zeta(r, \beta(r))-\zeta\left(r_{0}, \beta_{0}\right)}{2\left(r-r_{0}\right)}=\operatorname{det}\left({\overline{J_{F}\left(z_{0}\right)}}^{\prime} J_{F}\left(z_{0}\right)\right) \frac{(n+1) r_{0}+\operatorname{Tr}\left(D^{2} F(0) u_{0}\right)}{1-r_{0}^{2}}+O\left(\left|r-r_{0}\right|\right)$.

As $r \rightarrow r_{0}$, the limit of the left hand side of (3.10) is $\frac{1}{2}\left[\zeta_{r}+\beta^{\prime} \varphi_{\beta}\right]_{r=r_{0}}$. We evaluate it.

Noting that

$$
\zeta_{r}=\frac{\partial}{\partial r} \frac{(1+r)^{2 \beta-(n+1)}}{(1-r)^{2 \beta+(n+1)}}=\frac{(1+r)^{2 \beta-n-2}[4 \beta+(2 \beta+n+1) r]}{(1-r)^{2 \beta+n+2}},
$$

we have

$$
\left.\zeta_{r}\right|_{r=r_{0}}=\operatorname{det}\left({\overline{J_{F}\left(z_{0}\right)}}^{\prime} J_{F}\left(z_{0}\right)\right) \frac{4 \beta_{0}+(2 n+2) r_{0}}{1-r_{0}^{2}}
$$

by (3.8). Moreover,

$$
\beta^{\prime} \varphi_{\beta}=2 \beta^{\prime}(r) \frac{(1+r)^{2 \beta-(n+1)}}{(1-r)^{2 \beta+(n+1)}} \log \frac{1+r}{1-r}
$$

we have

$$
\left.\beta^{\prime} \varphi_{\beta}\right|_{r=r_{0}}=2 \beta\left(r_{0}\right) \log \frac{1+r_{0}}{1-r_{0}} \operatorname{det}\left({\overline{J_{F}\left(z_{0}\right)}}^{\prime} J_{F}\left(z_{0}\right)\right)
$$

by (3.8). Thus

$$
\frac{1}{2}\left[\zeta_{r}+\beta^{\prime} \zeta_{\beta}\right]_{r=r_{0}}=\left(\frac{2 \beta_{0}+(n+1) r_{0}}{1-r_{0}^{2}}+\beta^{\prime}\left(r_{0}\right) \log \frac{1+r_{0}}{1-r_{0}}\right) \operatorname{det}\left(\overline{J_{F}\left(z_{0}\right)} J_{F}\left(z_{0}\right)\right) .
$$


As $r \rightarrow r_{0},(3.10)$ becomes the following equality

$$
\begin{aligned}
& \left(\frac{2 \beta_{0}+(n+1) r_{0}}{1-r_{0}^{2}}+\beta^{\prime}\left(r_{0}\right) \log \frac{1+r_{0}}{1-r_{0}}\right) \operatorname{det}\left({\overline{J_{F}\left(z_{0}\right)}}^{\prime} J_{F}\left(z_{0}\right)\right) \\
= & \frac{(n+1) r_{0}+\operatorname{Tr}\left(D^{2} F(0) u_{0}\right)}{1-r_{0}^{2}} \operatorname{det}\left(\overline{J_{F}\left(z_{0}\right)} J_{F}\left(z_{0}\right)\right)
\end{aligned}
$$

by (3.11). After cancel the term $\frac{(n+1) r_{0}}{1-r_{0}^{2}} \operatorname{det}\left({\overline{J_{F}\left(z_{0}\right)}}^{\prime} J_{F}\left(z_{0}\right)\right)$ on both of the provious equality, we have

$$
\begin{aligned}
& \left(\frac{2 \beta_{0}}{1-r_{0}^{2}}+\beta^{\prime}\left(r_{0}\right) \log \frac{1+r_{0}}{1-r_{0}}\right) \operatorname{det}\left(\overline{J_{F}\left(z_{0}\right)} J_{F}\left(z_{0}\right)\right) \\
= & \frac{\operatorname{Tr}\left(D^{2} F(0) u_{0}\right)}{1-r_{0}^{2}} \operatorname{det}\left({\overline{J_{F}\left(z_{0}\right)}}^{\prime} J_{F}\left(z_{0}\right)\right) .
\end{aligned}
$$

By the definition of $C(\mathcal{S})(1.19),\left|\operatorname{Tr}\left(D^{2} F(0) u_{0}\right)\right| \leq 2 C(\mathcal{S})$. Hence

$$
\begin{aligned}
& \left(\frac{2 \beta_{0}}{1-r_{0}^{2}}+\beta^{\prime}\left(r_{0}\right) \log \frac{1+r_{0}}{1-r_{0}}\right) \operatorname{det}\left({\overline{J_{F}\left(z_{0}\right)}}^{\prime} J_{F}\left(z_{0}\right)\right) \\
& \leq \frac{2 C(\mathcal{S})}{1-r_{0}^{2}} \operatorname{det}\left({\overline{J_{F}\left(z_{0}\right)}}^{\prime} J_{F}\left(z_{0}\right)\right)
\end{aligned}
$$

by (3.12). But $\beta_{0}>C(\mathcal{S})$, it implies $\beta^{\prime}\left(r_{0}\right)<0$. We may use the argument similar in the proof of Theorem 2.1. We prove that there are a sequence of number $\left\{r_{j}\right\}$ decreasing to zero and a sequence $\left\{F_{j}\right\} \subset \mathcal{S}$ such that

$$
\begin{aligned}
& \operatorname{det}\left(\overline{J_{F_{j}\left(z_{j}\right)}} J_{F_{j}}\left(z_{j}\right)\right) \\
> & \frac{\left(1+r_{j}\right)^{2 \beta_{0}-(n+1)}}{\left(1-r_{j}\right)^{2 \beta_{0}+(n+1)}} \\
= & \left(1+(2 \beta-n-1) r_{j}+\cdots \cdots\right)\left(1-(2 \beta+n+1) r_{j}+\cdots \cdots\right)^{-1} \\
= & 1+4 \beta_{0} r_{j}+O\left(r_{j}^{2}\right)
\end{aligned}
$$

where $z_{j}=r_{j} u_{j}, u_{j} \in \partial \boldsymbol{B}^{n}, j=1,2, \cdots \cdots$.

We know that

$$
\operatorname{det}\left({\overline{J_{F_{j}}\left(z_{j}\right)}}^{\prime} J_{F_{j}}\left(z_{j}\right)\right)=1+2 \operatorname{Tr}\left[\Re e\left(D^{2} F_{j}(0) z_{j}\right)\right]+O\left(r_{j}^{2}\right) .
$$

Thus

$$
2 \operatorname{Tr}\left[\Re e\left(D^{2} F_{j}(0) z_{j}\right)\right] \geq 4 \beta_{0} r_{j}+O\left(r_{j}^{2}\right), \quad j=1,2, \cdots \cdots,
$$

by (3.13). When $C(\mathcal{S})$ is finite, then $\|$ ord $\| \mathcal{S}$ is finite, and $\mathcal{S}$ is a normal family. There is a subsequence of $\left\{F_{j}\right\}$ that converges locally uniformly to mapping $f \in \mathcal{S}$ and $u_{j}$ tend to a unit vector $u$. Thus dividing by $r_{j}$ on both sides of (3.14) and letting $j \rightarrow \infty$ through the subsequence, we obtain

$$
\left|\operatorname{Tr}\left(D^{2} F(0) u\right)\right| \geq 2 \beta_{0}>2 C(\mathcal{S}) .
$$

This contradiction complete the proof of the upper bound inequality in Theorem 3.1.

The proof of the lower bound inequality in Theorem 3.1 is similar with the proof of upper bound. We omit the detail of the proof. 
4. Covariant derivative of biholomorphic convex mappings on unit ball. In Section 1, we already mentioned that (1.9) has many consequences, e.g., (1.11), (1.12), (1.13) and (1.14) are its consequences. In this section, we will give another consequence. It is about the covariant derivative of biholomorphic convex mappings on unit ball $\boldsymbol{B}^{n}$ in $\mathbb{C}^{n}$. In this section, all vectors are row vectiors.

THEOREM 4.1. Let $f(z)$ be a normalized biholomorphic convex mapping on the unit ball $\boldsymbol{B}^{n}$ in $\mathbb{C}^{n}$, then for every $z \in \boldsymbol{B}^{n}$ and every vector $\eta(z)=\left(\eta_{1}(z), \cdots \cdots, \eta_{n}(z)\right)$ $\in \mathbb{C}^{n}$, the inequality

$$
\left|\left(\eta^{[k]} \cdot\left(\frac{\delta}{\delta z}\right)^{[k]}\right) f(z)\right| \leq k ! \frac{1+|z|}{1-|z|}\left|\eta \frac{R}{\sqrt{1-z \bar{z}}}\right|^{k}
$$

holds, where $\frac{\delta}{\delta z}=\left(\frac{\delta}{\delta z_{1}}, \cdots \cdots, \frac{\delta}{\delta z_{n}}\right)^{\prime}$ is the covariant derivative operator with respect to the Bergman metric of $\boldsymbol{B}^{n}, \xi^{[k]}$ means $k$-th Kronecker product of vector $\xi \in \mathbb{C}^{n}$ and

$$
R(z)=-I-\frac{\bar{z}^{\prime} z\left(1-\sqrt{1-z \bar{z}^{\prime}}\right)}{z \bar{z}^{\prime} \sqrt{1-z \bar{z}^{\prime}}}
$$

$I$ is the identity matrix.

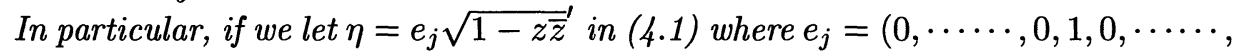
$0)$ is the unit vector of $j-$ th coordinate, we have the estimation of $k-t h$ covariant derivative of $f$ with respect to $z_{j}, j=1,2, \cdots \cdots, n ; k=1,2, \cdots \cdots$.

COROLlARY 4.2. Let $f(z)$ be a normalized biholomorphic convex mapping on the ball $\boldsymbol{B}^{n}$ in $\mathbb{C}^{n}$, then for every $z \in \boldsymbol{B}^{n}$, the inequality

$$
\left|\frac{\delta^{k}}{\delta z_{j}^{k}} f(z)\right| \leq \frac{k !\left(1-|z|^{2}+\left|z_{j}\right|^{2}\right)^{k / 2}}{(1+|z|)^{k-1}(1-|z|)^{k+1}}
$$

holds for $j=1,2, \cdots \cdots, n ; k=1,2, \cdots \cdots$.

From (1.9), it is easy to verify

$$
\frac{\left(1-|z|^{2}+\left|z_{j}\right|^{2}\right)^{\frac{1}{2}}}{(1+|z|)^{2}} \leq\left|\frac{\partial f}{\partial z_{j}}\right| \leq \frac{\left(1-|z|^{2}+\left|z_{j}\right|^{2}\right)^{\frac{1}{2}}}{(1-|z|)^{2}}, j=1,2, \cdots \cdots, n
$$

holds.

When $k=1$ in (4.3), it is the right hand side inequality of (4.4).

When $n=1,(4.3)$ is coincide with a result in [3].

If we let $\eta=z \sqrt{1-z \bar{z}^{\prime}}$ in (4.1), we have the following

COROLlaRY 4.3. Let $f(z)$ be a normalized biholomorphic convex mapping on the unit ball $\boldsymbol{B}^{n}$ in $\mathbb{C}^{n}$, then for every $z \in \boldsymbol{B}^{n}$, the inequality

$$
\left|\left(z^{[k]} \cdot\left(\frac{\delta}{\delta z}\right)^{[k]}\right) f(z)\right| \leq \frac{k !|z|^{k}}{(1+|z|)^{k-1}(1-|z|)^{k+1}}
$$

holds for $k=1,2, \cdots \cdots$.

Proof of Theorem 4.1. Gong and Yan [7] proved the following result.

Let $f(z)$ be a holomorphic mapping in $\boldsymbol{B}^{n}$, and $z=\varphi_{a}(w) \in \operatorname{Aut}\left(\boldsymbol{B}^{n}\right)$ which maps $z=a$ to $w=0$. We may express the Taylor expansion of $f\left(\varphi_{a}(w)\right)$ at $w=0$ as 


$$
F(w)=f\left(\varphi_{a}(w)\right)=\sum_{k=0}^{\infty} \frac{1}{k !}\left(1-a \bar{a}^{\prime}\right)^{\frac{k}{2}}\left(w R^{-1}\right)^{[k]} \cdot\left(\frac{\delta}{\delta a}\right)^{[k]} f(a)
$$

where $R=R(a)$ is defined by (4.2) and $\left(\frac{\delta}{\delta a}\right)^{[k]} f(a)$ means the column vector $\left(\left.\left(\frac{\delta}{\delta z}\right)^{[k]} f(z)\right|_{z=a}\right)^{\prime}$

Normalizing the mapping $F(w)$, we have

$$
\begin{aligned}
& \frac{F(w)-F(0)}{\sqrt{1-a \bar{a}}}\left(J_{f}(a)\right)^{-1} R \\
& =w+\sum_{k=2}^{\infty} \frac{1}{k !}\left(\sqrt{1-a \bar{a}^{\prime}}\right)^{k-1}\left(w R^{-1}\right)^{[k]} \cdot\left(\frac{\delta}{\delta a}\right)^{[k]} f(a)\left(J_{f}(a)\right)^{-1} R .
\end{aligned}
$$

It is a normalized biholomorphic convex mapping on $\boldsymbol{B}^{n}$ when $f(z)$ is a normalized biholomorphic convex mapping on $\boldsymbol{B}^{n}$.

We know that if $f(z)$ is a normalized biholomorphic convex mapping on $\boldsymbol{B}^{n}$, we may express it as

$$
f(z)=z+\sum_{m=2}^{\infty} P_{m}(z)
$$

where $P_{m}(z)=\left(P_{m}^{(1)}(z), \cdots \cdots, P_{m}^{(n)}(z)\right)$, and $P_{m}^{(j)}(z), j=1,2, \cdots \cdots, n$ are homogeneous polynomial of the elements of $z$ of degree $m$.

Gong and Liu [4] proved that

$$
\left|P_{m}(z)\right| \leq|z|^{m}, \quad m=2,3, \cdots \cdots,
$$

holds true for any $z \in \boldsymbol{B}^{n}$ if $f(z)$ is a normalized biholomorphic convex mapping on $B^{n}$.

From (4.5) and (4.6), we have that for any $w \in \boldsymbol{B}^{n}$,

$$
\frac{\left(\sqrt{1-a \bar{a}^{\prime}}\right)^{k-1}}{k !}\left|\left(w R^{-1}\right)^{[k]} \cdot\left(\frac{\delta}{\delta a}\right)^{[k]} f(a)\left(J_{f}(a)\right)^{-1} R\right| \leq|w|^{k}
$$

holds for all $k=2,3, \cdots \cdots$.

It is easy to verify that

$$
R \bar{R}^{\prime}=\frac{\bar{a}^{\prime} a+\left(1-a \bar{a}^{\prime}\right) I}{1-a \bar{a}^{\prime}} .
$$

Thus (4.7) is equivalent to

$$
\begin{aligned}
\left(w R^{-1}\right)^{[k]} \cdot\left(\frac{\delta}{\delta a}\right)^{[k]} f(a)\left(J_{f}(a)\right)^{-1} \frac{\bar{a}^{\prime} a+\left(1-a \bar{a}^{\prime}\right) I}{1-a \bar{a}^{\prime}} & \\
\cdot \overline{\left(J_{f}(a)\right)^{-1}}{ }^{\prime}\left(w R^{-1}\right)^{[k]} \cdot\left(\frac{\delta}{\delta a}\right)^{[k]} f(a) & \leq \frac{(k !)^{2}|w|^{2} k}{\left(1-a \bar{a}^{\prime}\right)^{k-1}} .
\end{aligned}
$$

From inequlities (1.9), we have

$$
\left(J_{f}(a)\right)^{-1}\left(\bar{a}^{\prime} a+\left(1-a \bar{a}^{\prime}\right) I\right){\overline{J_{f}(a)}}^{\prime-1} \geq(1-|a|)^{4} I .
$$


Applying (4.9) to (4.8), we obtain

$$
\left(w R^{-1}\right)^{[k]} \cdot\left(\frac{\delta}{\delta a}\right)^{[k]} f(a) \overline{\left(w R^{-1}\right)^{[k]} \cdot\left(\frac{\delta}{\delta a}\right)^{[k]} f(a)} \leq \frac{(k !)^{2}|w|^{2 k}}{\left(1-a \bar{a}^{\prime}\right)^{k-2}(1-|a|)^{4}}
$$

That is,

$$
\left|\left(w R^{-1}\right)^{[k]} \cdot\left(\frac{\delta}{\delta a}\right)^{[k]} f(a)\right| \leq \frac{k !|w|^{k}}{(1-|z|)^{2}\left(\sqrt{1-a \bar{a}^{\prime}}\right)^{k-2}} .
$$

Let $w=\eta R(a)$ in $(4.10)$, we get

$$
\left|\eta^{[k]} \cdot\left(\frac{\delta}{\delta a}\right)^{[k]} f(a)\right| \leq \frac{k !|\eta R|^{k}}{(1-|a|)^{2}\left(\sqrt{1-a \bar{a}^{\prime}}\right)^{k-2}}=k ! \frac{1+|a|}{1-|a|}\left|\frac{\eta R}{\sqrt{1-a \bar{a}^{\prime}}}\right|^{k}
$$

when $\eta R(a) \in \boldsymbol{B}^{n}$. Both sides of (4.11) are homogeneous polynomials of the elements of $\eta$ of degree $k$, thus (4.11) holds true for any $\eta \in \mathbb{C}^{n}$.

Replace $a$ by $z$ at (4.11), we have proved Theorem 4.1 .

\section{REFERENCES}

[1] R. W. Barnard, C. H. FitzGerald, ANd S. Gong, Distortion theorem for biholomorphic mappings in $\mathbb{C}^{2}$, Tran. Amer. Math. Soc., 344 (1994), pp. 907-924.

[2] S. Gong, Convex and Starlike Mappings in Several Complex Variables, Science Press/Kluwer Academic Publisher, 1998.

[3] S. Gong, The Bieberbach Conjecture, AMS/International Press, 1999.

[4] S. Gong AND T. S. LIU, The growth theorem of biholomorphic convex mappings on $\boldsymbol{B}_{p}$, Chin. Quar. Jour. of Math., 6 (1991), pp. 78-82.

[5] S. Gong AND T. S. LIU, Distortion theorems for biholomorphic convex mappings on bounded circular convex domains, Chin. Ann. of Math., 20B (1999), pp. 297-304.

[6] S. Gong, S. K. WANG, AND Q. H. YU, Biholomorphic convex mappings of ball in $\mathbb{C}^{n}$, Pacific Jour. of Math., 161 (1993), pp. 287-306.

[7] S. Gong AND Z. M. YAN, A note on Möbius transformations III, Chin. Quar. Jour. of Math., 1 (1986), pp. 33-40.

[8] S. Gong AND X. A. Zheng, Distortion theorem for biholomorphic mappings in transitive domains I, International Symposium in memory of L. K. Hua, Vol. II, Springer-Verlag, 1991, pp. 111-122.

[9] H. HAMADA AND G. KoHR, Linear invariant families on the unit polydisc, Preprint, 2000.

[10] L. Lempert, La metrique Kobayashi et las representation des domains sur la boule, Bull. Soc. Math. France, 109 (1981), pp. 427-474.

[11] Taishun LiU, Doctor Thesis, University of Sciece and Technology of China, 1989.

[12] J. A. Pfaltzgraff, Distortion of locally biholomorphic maps of the $n$-ball, Complex Variables, 33 (1997), 239-253.

[13] J. A. Pfaltzgraff AND T. S. Suffridge, Linear invariant order and convex maps in $\mathbb{C}^{n}$, Complex Variables, to appear.

[14] J. A. Pfaltzgraff AND T. S. Suffridge, Norm order and geometric properties of holomorphic mappings in $\mathbb{C}^{n}$, Preprint, 1999. 
